\title{
An interpolation theorem for slice-regular functions with application to very tame sets and slice Fatou-Bieberbach domains in $\mathbb{H}^{2}$
}

\section{Interpolation theorem and slice Fatou-Bieberbach domains in $\mathbb{\boxplus}^{2}$}

\author{
Jasna Prezelj ${ }^{1,2,3} \cdot$ Fabio Vlacci $^{4}$ (I) \\ Received: 15 March 2021 / Accepted: 14 January 2022 / Published online: 4 March 2022 \\ (c) The Author(s) 2022, corrected publication 2022
}

\begin{abstract}
We prove an interpolation theorem for slice-regular quaternionic functions. We define very tame sets in $\mathbb{T}^{2}$ to be the sets which can be mapped by compositions of automorphisms with volume 1 to the set $\mathcal{T}=\{(2 n-1,0), n \in \mathbb{N}\} \cup\{(2 n+\mathbb{S}, 0), n \in \mathbb{N}\}$. We then show that any zero set of an entire slice-regular function of one variable embedded in $\mathbb{H} \times\{0\} \subset \mathbb{H}^{2}$ is very tame in $\mathbb{W}^{2}$. A notion of slice Fatou-Bieberbach domain in $\mathbb{H}^{2}$ is introduced and, finally, a slice Fatou-Bieberbach domain in $\mathbb{H}^{2}$ avoiding $\mathcal{T}$ is constructed in the last section.
\end{abstract}

Keywords Quaternions · Slice-regular functions · Very tame sets · Slice Fatou-Bieberbach domain

Mathematics Subject Classification $30 \mathrm{G} 35 \cdot 41 \mathrm{~A} 05$

The first author was partially supported by research program P1-0291 and by research project J1-9104 at Slovenian Research Agency. Part of the paper was written when the first author was visiting the DiMaI at University of Florence and she wishes to thank this institution for its hospitality. The second author was partially supported by Progetto MIUR di Rilevante Interesse Nazionale PRIN 2010-2011 Varietà reali e complesse: geometria, topologia e analisi armonica. The research that led to the present paper was partially supported by a grant of the group GNSAGA of Istituto Nazionale di Alta Matematica 'F: Severi'

Fabio Vlacci

fvlacci@units.it

Jasna Prezelj

jasna.prezelj@fmf.uni-lj.si

1 UL FMF Jadranska 19, Ljubljana, Slovenia

2 UP FAMNIT Glagoljaška 8, Koper, Slovenia

3 IMFM Jadranska 19, Ljubljana, Slovenia

4 DiSPeS Università di Trieste, Piazzale Europa 1, Trieste, Italy 


\section{Introduction}

It is known that zero sets of slice-regular functions consist of points and spherical zeroes, namely isolated two-dimensional spheres of the form $S(a, r)=a+\mathbb{S} r:=\left\{z,(z-a)^{2}+r^{2}=0\right\}$ with $a \in \mathbb{R}, r>0$; the symbol $\mathbb{S}$ denotes the sphere of imaginary units in $\mathbb{U}$, namely $\mathbb{S}:=\left\{I \in \mathbb{U}, I^{2}=-1\right\}$. The sphere $S(a, r)$ is the zero set of the quadratic polynomial $(q-a)^{2}+r^{2}$. Zero sets of entire slice-regular functions consist of a discrete set of points and a 'discrete' set of spherical zeroes $S\left(a_{n}, r_{n}\right), n \in M \subset \mathbb{N}$ in the sense that the set $a_{n}^{2}+r_{n}^{2}, n \in M$ does not have accumulation points (see Theorem 3.21 in [3]). By the quaternionic version of the Weierstrass theorem proved in [5] we know, that any such a set, i.e., a union of a discrete set of points and a discrete set of isolated spheres as described above, is indeed a zero set of a slice-regular function. Since images of spheres $S\left(a_{n}, r_{n}\right)$ by slice-regular functions are no longer spheres, but rather generalized spheres of the form $S(q, \rho)=q+\mathbb{S} \rho$ with $q, \rho \in \mathbb{U}$, the corresponding interpolation theorem for quaternionic functions is about assigning values to points and generalized spheres to spheres. We prove such a theorem in Sect. 2.

In Sect. 3 we generalize the complex-analytic notion of very tame discrete set to a quaternionic setting. In the complex-analytic case, a tame set in $\mathbb{C}^{2}$ is any set which can be mapped to a subset of the set $\mathbb{N} \times\{0\} \subset \mathbb{C}^{2}$ by an automorphism of $\mathbb{C}^{2}$. If the automorphism can be chosen to have Jacobian equal to 1 , then the set is very tame (see [9]).

We have shown in [8] that the class of quaternionic automorphisms of $\mathbb{W}^{2}$ with volume (see Definition 5.1 in [8]) turns out to be a suitable counterpart in the quaternionic setting for holomorphic automorphisms of $\mathbb{C}^{2}$. Quaternionic automorphisms with volume 1 are the quaternionic analogues for holomorphic automorphisms with Jacobian equal to 1 (for the definition of quaternionic Jacobian see Sect. 1.1).

With this in mind we define (Definition 3) a very tame set in $\mathbb{Z}^{2}$ to be a union of a discrete set of points and a set of isolated spheres which can be injectively mapped to a subset of the set

$$
\mathcal{T}=\{(2 n-1,0), n \in \mathbb{N}\} \cup\{(2 n+\mathbb{S}, 0), n \in \mathbb{N}\} \subset \mathbb{H}^{2}
$$

by a diffeomorphism of $\mathbb{H}^{2}$, which is a limit of a convergent sequence of quaternionic automorphisms with volume 1 , such that points are mapped to points and spheres to spheres.

Having the interpolation theorem at our disposal, we are then able to show that zero sets of slice-regular functions in $\mathbb{H}$ embedded in $\mathbb{H}^{2}$ are very tame sets in $\mathbb{H}^{2}$. Moreover, the quaternionic automorphisms with volume 1 involved are shears (see Sect. 1.1).

In Sect. 4, we define quaternionic slice Fatou-Bieberbach domains and prove that there exists an example of a slice Fatou-Bieberbach domain in $\mathbb{Z}^{2}$ which does not intersect $\mathcal{T}$. The domain is obtained as a domain of convergence of compositions of shears which are slice-regular in the sense of [6].

\subsection{Definitions, notation and basic tools}

The present paper relies on previous work done by the authors, contained in [8], where the reader can find all the necessary definitions and theorems used in this paper. For reader's convenience, we briefly recall here some of the definitions and notations.

By $\mathcal{S R}(D)$ we refer to all slice-regular quaternionic functions on a (usually symmetric slice) domain $D \subseteq \mathbb{H}$ as defined in [3]. If a domain is a ball with radius $R$ centered at 0 , i.e. $D=B^{4}(0, R) \subset \mathbb{H}$, then $\mathcal{S R}(D)$ consists of all power series with coefficients 
on the right, i.e., a series of the form $\sum z^{n} a_{n}$, converging in $D$. By $\mathcal{H}[z, w](\Omega), \Omega \subset \mathbb{M}^{2}$, we denote the set of all functions defined as quaternionic power series converging in $\Omega$ with no restriction on the position of the quaternionic coefficients. This space coincides with all real analytic power series in 8 real variables with values in $\mathbb{H}$, converging in $\Omega$. If the coefficients of the series are only on the right hand-side, then the class is denoted by $\mathcal{H}_{\text {rhs }}[z, w]$. The notation $\mathcal{H}^{n}[z, w](\Omega)$, with $\Omega$ a domain in $\mathbb{W}^{2}$, denotes the set of all mappings $\Psi(z, w): \Omega \rightarrow \mathbb{Q}^{n}$ with components in $\mathcal{H}[z, w](\Omega)$. The mappings of the form $\Phi(z, w)=(z, w)+f(z-\mu w)(\mu, 1)$ or $\Psi(z, w)=(z, w)+g(w-v z)(1, v)$ with $\mu, v \in \mathbb{R}$ and $f, g \in \mathcal{S R}(\mathbb{W})$ are called shears and are automorphisms with volume 1, see [8]. In particular any shear of the form $\Phi(z, w)=(z, w)+f(z)(0,1)$ will be called a vertical shear and, similarly, $\Psi(z, w)=(z, w)+g(w)(1,0)$ will be called a horizontal shear.

The (Lie) derivative of $f \in \mathcal{H}[z, w](\Omega)$ in the direction $(h, k)$ at $(z, w) \in \Omega$ is defined as

$$
\lim _{\mathbb{R} \ni t \rightarrow 0} \frac{f(z+t h, w+t k)-f(z, w)}{t}:=\mathcal{L}_{(h, k)} f(z, w) .
$$

In particular, we will use the notation

$$
\mathcal{L}_{(h, 0)} f(z, w)=\hat{\partial}_{z} f(z, w)[h], \quad \mathcal{L}_{(0, k)} f(z, w)=\widehat{\partial}_{w} f(z, w)[k]
$$

and call them, respectively, partial derivative of $f \in \mathcal{H}[z, w](\Omega)$ with respect to $z$ (applied to $h$ ) and partial derivative of $f \in \mathcal{H}[z, w](\Omega)$ with respect to $w$ (applied to $k$ ). The operators $\hat{\partial}_{z} f(z, w)[h]$ and $\hat{\partial}_{w} f(z, w)[h]$ are additive and real homogeneous in $h$. The Jacobian of the mapping $F=\left(f_{1}, f_{2}\right) \in \mathcal{H}^{2}[z, w](\Omega)$ applied to $\left(h_{1}, h_{2}\right)$ is defined to be

$$
D F(z, w)\left[h_{1}, h_{2}\right]:=\left[\begin{array}{ll}
\hat{\partial}_{z} f_{1}(z, w)\left[h_{1}\right] & \hat{\partial}_{w} f_{1}(z, w)\left[h_{2}\right] \\
\hat{\partial}_{z} f_{2}(z, w)\left[h_{1}\right] & \hat{\partial}_{w} f_{2}(z, w)\left[h_{2}\right]
\end{array}\right] .
$$

Notice that if $F \in \mathcal{H}^{2}[z, w]$, then

$$
F\left(z+h_{1}, w+h_{2}\right)-F(z, w)=D F(z, w)\left[h_{1}, h_{2}\right]\left[\begin{array}{l}
1 \\
1
\end{array}\right]+o\left(\left\|\left(h_{1}, h_{2}\right)\right\|\right)
$$

For the Jacobian, we use the matrix norm $|\cdot|_{\infty}$ defined by

$$
|D F(z, w)|_{\infty}=\max \left\{\left|D F(z, w)\left[h_{1}, h_{2}\right]\right|_{\infty},\left|h_{1}\right|,\left|h_{2}\right| \leq 1\right\},
$$

where

$$
\begin{gathered}
\left|D F(z, w)\left[h_{1}, h_{2}\right]\right|_{\infty}=\max \left\{\left|\hat{\partial}_{z} f_{1}(z, w)\left[h_{1}\right]+\hat{\partial}_{w} f_{1}(z, w)\left[h_{2}\right]\right|,\right. \\
\left.\left|\hat{\partial}_{z} f_{2}(z, w)\left[h_{1}\right]+\hat{\partial}_{w} f_{2}(z, w)\left[h_{2}\right]\right|\right\} .
\end{gathered}
$$

The adapted chain rule holds, namely

$$
D(F \circ G)(z, w)\left[h_{1}, h_{2}\right]=D F(G(z, w)) \diamond D G(z, w)\left[h_{1}, h_{2}\right],
$$

where $\diamond$ is defined by

$$
\begin{aligned}
& D(F \circ G)(z, w)\left[h_{1}, h_{2}\right] \\
& =\left[\begin{array}{ll}
\hat{\partial}_{z} f_{1}(u, v)\left[a_{1}\right]+\hat{\partial}_{w} f_{1}(u, v)\left[a_{2}\right] & \hat{\partial}_{z} f_{1}(u, v)\left[b_{1}\right]+\hat{\partial}_{w} f_{1}(u, v)\left[b_{2}\right] \\
\hat{\partial}_{z} f_{2}(u, v)\left[a_{1}\right]+\hat{\partial}_{w} f_{2}(u, v)\left[a_{2}\right] & \hat{\partial}_{z} f_{2}(u, v)\left[b_{1}\right]+\hat{\partial}_{w} f_{2}(u, v)\left[b_{2}\right]
\end{array}\right],
\end{aligned}
$$


with

$$
D F(u, v)=\left[\begin{array}{ll}
\hat{\partial}_{z} f_{1}(u, v) & \hat{\partial}_{w} f_{1}(u, v) \\
\hat{\partial}_{z} f_{2}(u, v) & \hat{\partial}_{w} f_{2}(u, v)
\end{array}\right] \text { and } D G(z, w)\left[h_{1}, h_{2}\right]=\left[\begin{array}{ll}
a_{1} & b_{1} \\
a_{2} & b_{2}
\end{array}\right] .
$$

It is immediate that the norm $|\cdot|_{\infty}$ is definite, right absolutely homogeneous and sub-additive. It is also sub-multiplicative for the operator $\diamond$, i.e.

$$
|D(F \circ G)(z, w)|_{\infty} \leq|D F(G(z, w))|_{\infty} \cdot|D G(z, w)|_{\infty} .
$$

To show this, assume first that $\left|D G(z, w)\left[h_{1}, h_{2}\right]\right|_{\infty}=\left|a_{1}+b_{1}\right|$, define

$$
k_{1}:=\frac{a_{1}+b_{1}}{\left|a_{1}+b_{1}\right|}, k_{2}:=\frac{a_{2}+b_{2}}{\left|a_{1}+b_{1}\right|}
$$

and notice that $\left|k_{1}\right|,\left|k_{2}\right| \leq 1$. To estimate the norm of the derivative we use additivity and real homogeneity of the operators $\hat{\partial}_{z}, \widehat{\partial}_{w}$ in the variables $h$ and $k$. We have

$$
\begin{aligned}
\left|D(F \circ G)(z, w)\left[h_{1}, h_{2}\right]\right|_{\infty}= & \max \left\{\left|\hat{\partial}_{z} f_{1}(u, v)\left[a_{1}\right]+\hat{\partial}_{w} f_{1}(u, v)\left[a_{2}\right]+\hat{\partial}_{z} f_{1}(u, v)\left[b_{1}\right]+\hat{\partial}_{w} f_{1}(u, v)\left[b_{2}\right]\right|,\right. \\
& \left.\left|\hat{\partial}_{z} f_{2}(u, v)\left[a_{1}\right]+\hat{\partial}_{w} f_{2}(u, v)\left[a_{2}\right]+\hat{\partial}_{z} f_{2}(u, v)\left[b_{1}\right]+\hat{\partial}_{w} f_{2}(u, v)\left[b_{2}\right]\right|\right\}, \\
= & \max \left\{\left|\hat{\partial}_{z} f_{1}(u, v)\left[a_{1}+b_{1}\right]+\hat{\partial}_{w} f_{1}(u, v)\left[a_{2}+b_{2}\right]\right|,\right. \\
& \left.\left|\hat{\partial}_{z} f_{2}(u, v)\left[a_{1}+b_{1}\right]+\hat{\partial}_{w} f_{2}(u, v)\left[a_{2}+b_{2}\right]\right|\right\} \\
= & \max \left\{\left|\hat{\partial}_{z} f_{1}(u, v)\left[k_{1}\right]+\hat{\partial}_{w} f_{1}(u, v)\left[k_{2}\right]\right|,\left|\hat{\partial}_{z} f_{2}(u, v)\left[k_{1}\right]+\hat{\partial}_{w} f_{2}(u, v)\left[k_{2}\right]\right|\right\} . \\
& \cdot\left|a_{1}+b_{1}\right| \\
\leq & |D F(z, w)|_{\infty}\left|D G(z, w)\left[h_{1}, h_{2}\right]\right|
\end{aligned}
$$

and the same estimate $\left|D(F \circ G)(z, w)\left[h_{1}, h_{2}\right]\right|_{\infty} \leq|D F(z, w)|_{\infty}\left|D G(z, w)\left[h_{1}, h_{2}\right]\right|$ holds also if $\left|D G(z, w)\left[h_{1}, h_{2}\right]\right|_{\infty}=\left|a_{2}+b_{2}\right|$. Taking the max over $\left|h_{1}\right|,\left|h_{2}\right| \leq 1$ yields the desired estimate.

\section{The interpolation theorem for slice-regular functions}

As already mentioned in the Introduction, an interpolation theorem in the quaternionic setting should provide the existence of a quaternionic function which assigns prescribed values to points and prescribed generalized spheres to spheres. Let us begin by stating the formal definitions.

Definition 1 A generalized sphere in $\mathbb{W}$ is any set of the form $S(q, \rho)=q+\mathbb{S} \rho$, with $q, \rho \in \mathbb{H}$. If $q=a \in \mathbb{R}, \rho=r \in[0, \infty)$ we use the notation $S(a, r)$ and we call them spheres. In particular, a sphere becomes a real number when $r=0$.

Remark 1 Zero sets of entire slice-regular functions may contain only spheres besides isolated quaternions (see e.g., [4]). In this framework we consider the zero set of a slice-regular function as a geometric object, i.e., as a union of points where the slice-regular function $f$ vanishes. Algebraically, a point $z_{k}=a_{k}+I b_{k}$ may be an isolated zero of multiplicity $n$ in the sense that $f(z)=\left(z-z_{k}\right)^{* n} * f_{1}(z)$ and at the same time there may be a spherical zero $a_{k}+b_{k} S$, which contains $z_{k}$. We only consider the spherical zero in such cases for the purpose of the theorem. 
Definition 2 Let the zero set $Z$ of an entire slice-regular function be given. We define the decomposition of $Z$ to be $Z=\mathcal{S} \cup \mathcal{Z} \cup \mathcal{X}$, where $\mathcal{S}$ is the discrete set of spheres, $\mathcal{S}:=\left\{S_{j}=S\left(a_{j}, r_{j}\right), j \in M_{1} \subset \mathbb{N}, r_{j}>0\right\}, \mathcal{Z}$ is the discrete set of non-real zeroes, $\mathcal{Z}:=\left\{z_{k} \in \mathbb{U} \backslash \mathbb{R}, k \in M_{2} \subset \mathbb{N}\right\}$ and $\mathcal{X}$ is the discrete set of real zeroes, $\mathcal{X}:=\left\{x_{l} \in \mathbb{R}, l \in M_{3} \subset \mathbb{N}\right\}$.

Theorem 1 (The Interpolation Theorem). Let the zero set $Z$ of an entire slice-regular function be given with the decomposition $Z=\mathcal{S} \cup \mathcal{Z} \cup \mathcal{X}$ as in Definition 2. Assume that $z_{k} \notin S\left(a_{j}, r_{j}\right)$ for any $j, k$. For any sequence of generalized spheres $\breve{S}_{j}=S\left(q_{j}, \rho_{j}\right), j \in M_{1}$ and values $w_{k}, u_{l} \in \mathbb{H}, k \in M_{2}, l \in M_{3}$, there exists an entire slice-regular function $f$ such that $f\left(S\left(a_{j}, r_{j}\right)\right)=S\left(q_{j}, \rho_{j}\right), f\left(z_{k}\right)=w_{k}$ and $f\left(x_{l}\right)=u_{l}$ for all $j \in M_{1}, k \in M_{2}$ and $l \in M_{3}$.

By the assumption on discreteness of the spheres $S_{j}$, the sequence $R_{j}^{2}=a_{j}^{2}+r_{j}^{2}$ has no accumulation point. We may assume that it is (not necessarily strictly) increasing. The main ingredient for the proof of the Theorem is the following

Lemma 1 Let the set $Z$ and the sets $S_{j}, z_{k}$ and $x_{l}$ be as in Theorem 1 . Choose $j_{0} \in M_{1}, \varepsilon>0$ and let $0<R<R_{j_{0}}=\left(a_{j_{0}}^{2}+r_{j_{0}}^{2}\right)^{1 / 2}$. For any generalized sphere $\check{S}=S(q, \rho)$ there exists a slice-regular function $f_{j_{0}}$ such that $f_{j_{0}}\left(S_{j_{0}}\right)=\check{S}, f_{j_{0}}$ vanishes on the set $Z \backslash S_{j_{0}}$ and $\left|f_{j_{0}}\right|_{B^{4}(0, R)}<\varepsilon$. If all the generalized spheres are spheres, then the function $f_{j_{0}}$ can be chosen to be slice-preserving, i.e., such that it has a power series expansion with real coefficients.

Proof For any point $z_{k} \in \mathcal{Z}$, we can consider the corresponding sphere $\tilde{S}_{k} \ni z_{k}$. By assumption, also the spheres $\tilde{S}_{k}$ are discrete; namely if $z_{k}=a_{k}+I_{k} r_{k} \in S\left(a_{k}, r_{k}\right)=\tilde{S}_{k}$, then $\tilde{S}_{k} \cap S_{j}=\emptyset$ for any $S_{j} \in \mathcal{S}$. By the quaternionic version of the Weierstrass theorem as in [5], there exists a slice-preserving function $g$ with zeroes precisely on $S_{j} \neq S_{j_{0}}, \tilde{S}_{k}$ and $x_{l}$ so the coefficients of its power series are real. Therefore the image of the sphere $S_{j_{0}}$ is again a sphere, $g\left(S_{j_{0}}\right)=S(a, r)$, more precisely, $g\left(a_{j_{0}}+I r_{j_{0}}\right)=a+I r$ with $a^{2}+r^{2} \neq 0$. In order to satisfy also the condition $f\left(S_{j_{0}}\right)=\breve{S}$, we are looking for a slice-regular function $\gamma(z)=z \alpha+\beta$ such that

$$
g\left(a_{j_{0}}+I r_{j_{0}}\right) \gamma\left(a_{j_{0}}+I r_{j_{0}}\right)=q+I \rho .
$$

Since $\gamma\left(a_{j_{0}}+I r_{j_{0}}\right)=a_{j_{0}} \alpha+\beta+I r_{j_{0}} \alpha$, equation (1) implies

$$
a_{j_{0}} \alpha+\beta+I r_{j_{0}} \alpha=\frac{1}{a^{2}+r^{2}}(a-I r)(q+I \rho)=\frac{1}{a^{2}+r^{2}}(a q+r \rho+I(a \rho-r q))
$$

and so we get

$$
\alpha=\frac{a \rho-r q}{r_{j_{0}}\left(a^{2}+r^{2}\right)}, \quad \beta=\frac{a q+r \rho}{a^{2}+r^{2}}-a_{j_{0}} \alpha .
$$

Hence $g \cdot \gamma$ is a slice-regular function which also satisfies the interpolation condition $(g \cdot \gamma)\left(S_{j_{0}}\right)=\check{S}$. For the statement of the theorem about approximation, observe that any given sphere $S_{j}=\left\{a_{j}+r_{j} I, I \in \mathbb{S}\right\}$ uniquely determines $\vartheta_{j} \in(0,1)$ such that $a_{j}+r_{j} I=R_{j} e^{I \pi \vartheta_{j}}$ for any point $a_{j}+r_{j} I \in S_{j}$. If $\vartheta_{j}$ is a rational number, then for some $n \in \mathbb{N}$ we have $\left(\left(a_{j}+r_{j} I\right) / R_{j}\right)^{n}=1$ and so the requested estimate on the ball $B^{4}(0, R)$ is obtained if we multiply $g \cdot \gamma$ by $\left(z / R_{j_{0}}\right)^{N n}$ for $N$ large enough. In other words, the function 


$$
f_{j_{0}}(z)=\left(\frac{z}{R_{j_{0}}}\right)^{N n} g(z) \gamma(z)
$$

has all the required properties. If $\vartheta_{j}$ is not rational, then the set of points $\left\{\left(\left(a_{j}+r_{j} I\right) / R_{j}\right)^{n}\right.$, $n \in \mathbb{N}\}$ is dense in the unit circle $S_{I}^{1}$ of $\mathbb{C}_{I}$. Therefore, for some increasing sequence of natural numbers $\left\{n_{k}\right\}_{k \in \mathbb{N}}$, we have

$$
\left(\frac{a_{j_{0}}+r_{j_{0}} I}{R_{j_{0}}}\right)^{n_{k}}=c_{n_{k}}-d_{n_{k}} I \rightarrow \frac{a_{j_{0}}-r_{j_{0}} I}{R_{j_{0}}} .
$$

Define $\alpha_{n_{k}}, \beta_{n_{k}} \in \mathbb{R}$ to be such that $c_{n_{k}}+d_{n_{k}} I=\alpha_{n_{k}}\left(a_{j_{0}}+r_{j_{0}} I\right)+\beta_{n_{k}}$. Since by (3) we have $c_{n_{k}}+d_{n_{k}} I \approx\left(a_{j_{0}}+r_{j_{0}} I\right) / R_{j_{0}}$, it is clear that $\alpha_{n_{k}} \approx 1 / R_{j_{0}}$ and $\beta_{n_{k}} \approx 0$. Hence, we can find a sequence of functions $\psi_{n_{k}}(z)=\alpha_{n_{k}} z+\beta_{n_{k}}$ with $\alpha_{n_{k}}, \beta_{n_{k}} \in \mathbb{R}$, such that

$$
\left(\frac{z}{R_{j_{0}}}\right)^{n_{k}} \psi_{n_{k}}(z)=1 \text { for any } z \in S_{j_{0}} \text { and } \psi_{n_{k}}(z) \rightarrow z / R_{j_{0}} \text {. }
$$

Furthermore, if $z \in B^{4}(0, R) \subset \mathbb{H}$, for any positive $\varepsilon$, there exists $k_{0}$ such that for any $k>k_{0}$ the estimate

$$
\left|\left(\frac{z}{R_{j_{0}}}\right)^{n_{k}} \psi_{n_{k}}(z) g(z) \gamma(z)\right|<\varepsilon
$$

holds. Then the function $f$ defined by

$$
f_{j_{0}}(z):=\left(\frac{z}{R_{j_{0}}}\right)^{n_{k}} \psi_{n_{k}}(z) g(z) \gamma(z)
$$

for $k$ sufficiently large has all the desired properties.

Notice that if $q, \rho \in \mathbb{R}$, then the numbers $\alpha, \beta$ given by (2) are real, so $\gamma$ has real coefficients. Thus the last statement of the Lemma is also proven.

Lemma 2 Let the set $Z$ and the sequences $S_{j}, z_{k}$ and $x_{l}$ be as in Theorem 1. Choose $k_{0} \in M_{2}, \varepsilon>0$ and let $0<R<R_{k_{0}}=\left|z_{k_{0}}\right|$. For any $w \in \mathbb{U}$ there exists a slice-regular function $f_{k_{0}}$ such that $f_{k_{0}}\left(z_{k_{0}}\right)=w, f_{k_{0}}$ vanishes on $Z \backslash\left\{z_{k_{0}}\right\}$ and $\left|f_{k_{0}}\right|_{B^{4}(0, R)}<\varepsilon$. The same holds with $x_{l_{0}}$ in place of $z_{k_{0}}$.

Proof From [5] there exists an entire slice-regular function with zeroes precisely on $S_{j}, z_{k} \neq z_{k_{0}}$ and $x_{l}$ of the form

$$
g(z)=S(z) X(z) Z(z),
$$

where $S$ has precisely the union of spheres $S_{j}$ as zero set, $X$ has the union of the real numbers $x_{l}$ as zero set and $Z$ has the union of points $z_{k} \neq z_{k_{0}}$ as zero set. Define $\lambda=g\left(z_{k_{0}}\right), \lambda \neq 0$ by the assumptions above, and let $u=w^{-1} z_{k_{0}} w$. Then for sufficiently large $N$ the function

$$
f_{k_{0}}(z)=z^{N} g(z) \lambda^{-1} w u^{-N}
$$

fulfills all the requested conditions of the Lemma. 
Proof of the Interpolation Theorem We are looking for a function $f$ of the form $f=F_{1}+F_{2}+F_{3}$, where $F_{1}, F_{2}, F_{3}$ are slice-regular functions such that

1. $F_{1}\left(S_{j}\right)=\check{S}_{j}$ for $j \in M_{1}$ and $\left.F_{1}\right|_{\mathcal{Z} \cup \mathcal{X}} \equiv 0$

2. $\quad F_{2}\left(z_{k}\right)=w_{k}$ for $k \in M_{2}$ and $\left.F_{2}\right|_{\mathcal{S} \cup \mathcal{X}} \equiv 0$

3. $\quad F_{3}\left(x_{l}\right)=u_{l}$ for $l \in M_{3}$ and $\left.F_{3}\right|_{\mathcal{S} \cup \mathcal{Z}} \equiv 0$.

For the construction of $F_{1}$ observe that if the set $M_{1}$ is finite then we can simply define the function $F_{1}(z):=\sum_{j \in M_{1}} f_{j}$ with $f_{j}$ given by Lemma 1 since the sum is finite.

Now assume that the sequence of spheres is infinite and that the corresponding radii $R_{j}$ form an increasing sequence. If there are $n_{j}$ spheres with the same radius indexed from $j_{n}$ to $j_{n}+n_{j}-1$, namely $R_{j_{n}}=\ldots=R_{j_{n}+n_{j}-1}$, we require that functions $f_{j}, j=j_{n}, \ldots j_{n}+n_{j}-1$ satisfy the estimate $\left|f_{j}(z)\right|<2^{-j}$ on $B\left(0, R_{j_{n}-1}\right)$. Then $F_{1}(z):=\sum_{j \in M_{1}} f_{j}$ converges, $F_{1}\left(S_{j}\right)=\check{S}_{j}$ for $j \in M_{1}$ and $F_{1}$ vanishes in $\mathcal{Z} \cup \mathcal{X}$.

The constructions of $F_{2}$ and $F_{3}$ are similar and rely upon Lemma 2 in the place of Lemma 1.

Lemmas 1 and 2 together with Theorem 1 imply the

Corollary 1 If the set $Z$ (as above) does not intersect the ball $B^{4}(0, r) \in \mathbb{U}$, then the interpolation function obtained in Theorem 1 can be chosen to be arbitrarily small on compact subsets of $B^{4}(0, r)$.

\section{Very tame sets in $\mathbb{\boxplus}^{2}$}

There are different notions of tameness in the literature and we refer the interested reader to [1] for an updated historical review on this subject. We only recall here that the notion of very tameness in complex analysis for a discrete set refers to correspondence via volume-preserving automorphisms (that is automorphisms with Jacobian equal to 1) with an embedded copy of $\mathbb{N}$.

Here we give the following

Definition 3 The standard tame set is defined as

$$
\mathcal{T}=\underbrace{\{(2 n-1,0), n \in \mathbb{N}\}}_{\mathcal{T}_{p}} \cup \underbrace{\{(2 n+\mathbb{S}, 0), n \in \mathbb{N}\}}_{\mathcal{T}_{S}} .
$$

A very tame set in $\mathbb{H}^{2}$ is any discrete set $\mathfrak{T}=\mathfrak{I}_{p} \cup \mathfrak{I}_{S}$ of points $\left(\mathfrak{I}_{p}\right)$ and spheres $\left(\mathfrak{I}_{S}\right)$ which can be mapped injectively to a subset of $\mathcal{T}^{\prime} \subseteq \mathcal{T}$ by a diffeomorphism of $\mathbb{U}^{2}$, which is a limit of a convergent sequence of quaternionic automorphisms with volume 1 (in the sense of Definition 5.1 in [8]) such that points are mapped to points and spheres are mapped to spheres, i.e.

$$
\mathfrak{I}_{p} \cong \mathcal{T}_{p} \cap \mathcal{T}^{\prime} \text { and } \mathfrak{I}_{S} \cong \mathcal{T}_{S} \cap \mathcal{T}^{\prime}
$$


Remark 2 Recall that quaternionic automorphisms with volume 1 are generalizations of holomorphic automorphisms with Jacobian equal to 1; furthermore, any composition of holomorphic automorphisms with Jacobian equal to 1 is again a holomorphic automorphism with Jacobian equal to 1 . In the quaternionic setting a composition of two automorphisms with volume 1 is not necessarily an automorphism with volume.

The result which motivates the definition of a very tame set in the quaternionic setting and gives a geometric interpretation of the link with the theory of slice-regular function is the following

Theorem 2 The zero set $Z$ of any slice-regular function $f \in \mathcal{S R}(\mathbb{M}), f \not \equiv 0$ embedded in $\mathbb{W} \times\{0\}$ is a very tame set in $\mathbb{H}^{2}$. More precisely, the set $Z \times\{0\}$ can be mapped to $\mathcal{T}$ by a finite composition of slice-regular shears with only real coefficients in their power series expansions.

Proof We follow the idea of the proof presented in the paper [9] by Rosay and Rudin where vertical and horizontal shears are applied in order to find the 1-1 correspondence of points in $Z$ with the ones in $\mathbb{N} \times\{0\} \subset \mathbb{C}^{2}$. Geometrically, this is achieved by labelling points in $Z=\left\{z_{j}: j \in \mathbb{N}\right\}$ and using the vertical shear $\Phi_{1}(z, w)=\left(z, w+f_{1}(z)\right)$ such that $\Phi_{1}\left(z_{j}, 0\right)=\left(z_{j}, j\right)$ to lift them in $\mathbb{C}^{2}$ to different levels. Then, one considers the horizontal shear $\Psi(z, w)=(z+g(w), w)$ such that $\Psi\left(z_{j}, j\right)=(j, j)$ and then the verical shear $\Phi_{2}(z, w)=\left(z, w+f_{2}(z)\right)$ such that $\Phi_{2}(j, j)=(j, 0)$. Hence $\Phi_{2} \circ \Psi \circ \Phi_{1}$ is the desired composition of automorphisms.

We proceed similarly with the difference that we first find an automorphism which injectively maps the spheres of $Z$ to the spheres of $\mathcal{T}$ and leave the points of $Z$ fixed and, afterwards, find an automorphisms which injectively maps the points of $Z$ to the points of $\mathcal{T}$ and keeps the spheres of $\mathcal{T}$ fixed.

We may (and will) also assume that the zero set $Z$ contains infinitely many real and nonreal points and infinitely many spheres and that $(Z \times\{0\}) \cap \mathcal{T}=\emptyset$, otherwise we just translate the coordinate system a little bit in the real direction by a shear $\Psi_{0}(z, w)=(z+\varepsilon, w), \varepsilon \in \mathbb{R}$.

In the quaternionic setting, as a first step, we construct a composition of shears which maps spheres of $Z$ to spheres of $\mathcal{T}$ and leave fixed the real isolated zeroes and the spheres $\tilde{S}_{k}$ generated by non-real isolated zeroes. The idea of keeping fixed any entire sphere generated by a non-real zero in $Z$ allows us to consider shears in $\mathcal{H}_{\text {rhs }}^{2}$ whose restrictions to $\mathbb{C}_{I} \times \mathbb{C}_{I} \simeq \mathbb{C}^{2}$ for any $I \in \mathbb{S}$ can be regarded as complex holomorphic automorphisms with real coefficients in their power series expansions.

All spheres $S_{j}=S\left(a_{j}, r_{j}\right) \in Z$ and have to be mapped according to the following diagram

$$
\left(a_{j}+r_{j} I, 0\right) \stackrel{\Phi_{1}}{\longrightarrow}\left(a_{j}+r_{j} I, 2 j(1+I)\right) \stackrel{\Psi}{\longrightarrow}(2 j+I, 2 j(1+I)) \stackrel{\Phi_{2}}{\longrightarrow}(2 j+I, 0) .
$$

where $\Phi_{1}(z, w)=\left(z, w+f_{1}(z)\right)$ is the first vertical shear, $\Psi(z, w)=(z+g(w), w)$ is the horizontal shear and finally the shear $\Phi_{2}(z, w)=\left(z, w+f_{2}(z)\right)$ is the second vertical shear. The function $f_{1}$ in the first shear $\Phi_{1}$ has to be zero on all real points in $Z$ and spheres $\tilde{S}_{k}$ generated by non-real zeroes $z_{k} \in Z$, (i.e $f_{1}\left(\tilde{S}_{k}\right)=0$ for any $k$ and $f_{1}\left(x_{l}\right)=0$ for any $l$ ) and, in addition, $f_{1}\left(a_{j}+r_{j} I\right)=2 j(1+I)$ for all $j$; these interpolation conditions for $f_{1}$ can be fulfilled because, by assumption, $\mathcal{Z} \cap \mathcal{S}=\emptyset$. Next, the function $g$ has to leave the zero level $\mathbb{U} \times\{0\}$ fixed and move the spheres in $z$-direction so that they have the correct projection to $\mathbb{M} \times\{0\}$. In other words, the conditions for $g$ are 


$$
g(0)=0, \quad g(2 j(1+I))=-\left(a_{j}+r_{j} I\right)+2 j+I=-a_{j}+2 j+\left(-r_{j}+1\right) I \quad \forall j .
$$

At the end, using $\Phi_{2}$, we keep fixed the spheres $\tilde{S}_{k}$ and zeroes $x_{l}$ and map the spheres $\Psi \circ \Phi_{1}\left(S_{j}\right)$ to $(2 j+\mathbb{S}, 0) \in \mathcal{T}_{S}$ by imposing $f_{2}(2 j+I)=-2 j(1+I)$ and $f_{2}\left(\tilde{S}_{k}\right)=f_{2}\left(x_{l}\right)=0, \forall k, l$, which is possible because of the condition $(Z \times\{0\}) \cap \mathcal{T}=\emptyset$.

Notice that for this step the Interpolation Theorem is fundamental, since we have to assign spheres to given spheres, otherwise the map $g$ could not be defined. Moreover, since spheres are mapped to spheres, the coefficients in the power series expansions of $f_{1}, f_{2}$ and $g$ are real.

In the second step isolated zeroes are mapped to odd integers (we list all discrete zeroes in the sequence $y_{k}$ without distinguishing the real from the nonreal ones) and proceed as above by using new shears $\widetilde{\Phi}_{1}(z, w)=\left(z, w+\tilde{f}_{1}(z)\right), \widetilde{\Psi}=(z+\tilde{g}(w), w), \widetilde{\Phi}_{2}=\left(z, w+\tilde{f}_{2}(z)\right)$ according to the following diagram

$$
\left(y_{k}, 0\right) \stackrel{\widetilde{\Phi}_{1}}{\longrightarrow}\left(y_{k}, 2 k-1\right) \stackrel{\widetilde{\Psi}}{\longrightarrow}(2 k-1,2 k-1) \stackrel{\widetilde{\Phi}_{2}}{\longrightarrow}(2 k-1,0), k \in \mathbb{N},
$$

where the interpolation conditions for the functions $\tilde{f_{1}}, \tilde{f_{2}}, \tilde{g}$ are $\tilde{f}_{1}(2 j+\mathbb{S})=0, \tilde{f}_{1}\left(y_{k}\right)=$ $2 k-1, \tilde{g}(0)=0, \tilde{g}(2 k-1)=2 k-1-y_{k}, \tilde{f}_{2}(2 j+\mathbb{S})=\tilde{f}_{2}(0)=0, \tilde{f}_{2}(2 k-1)=-(2 k-1)$ for any $k, j$. Notice that the interpolation conditions for $\tilde{f}_{1}$ do not contradict each other because of the assumption that $(Z \times\{0\}) \cap \mathcal{T}=\emptyset$. Therefore $\widetilde{\Phi}_{2} \circ \widetilde{\Psi}_{\circ} \widetilde{\Phi}_{1} \circ \Phi_{2} \circ \Psi \circ \Phi_{1} \circ \Psi_{0}$ is the desired composition of shears which fulfills the correspondence of $Z$ with $\mathcal{T}$.

Remark 3 Observe that shears in the proof of Theorem 2 are in fact slice-regular mappings.

\section{Slice Fatou-Bieberbach domains in $\mathbb{\boxplus}^{2}$}

In this section we give a definition of slice Fatou-Bieberbach domains in $\mathbb{W}^{2}$ and then construct an example of a slice Fatou-Bieberbach domain in $\mathbb{W}^{2}$ which avoids the (standard) tame set $\mathcal{T}$. We recall that a Fatou-Bieberbach domain in $\mathbb{C}^{n}$ is a proper subset $\Omega \subset \mathbb{C}^{n}$, biholomorphic to $\mathbb{C}^{n}$. In the quaternionic setting the notion of analiticity is usually associated with the notion of slice-regulariwe can find a sequence ty. The class of slice-regular mappings is rather rigid, for example it is not preserved even by compositions with real linear transformations. This is the reason why in the next definition we require analiticity for the map $h: \mathbb{W}^{2} \rightarrow \Omega$, i.e., $h \in \mathcal{H}^{2}[z, w]$ (see Sect. 1.1 for the definition) but in addition we also require that the intersection of $\Omega$ with complex subspaces $\mathbb{C}_{I}^{2}=\mathbb{C}_{I} \times \mathbb{C}_{I}, I \in \mathbb{S}$, should coincide with the definition of a Fatou-Bieberbach domain in the complex setting.

Definition 4 A slice Fatou-Bieberbach domain in $\mathbb{T}^{2}$ is a proper subset $\Omega \subset \mathbb{Q}^{2}$ such that there exists a real analytic diffeomorphism $h: \mathbb{H}^{2} \rightarrow \Omega$, i.e., $h \in \mathcal{H}^{2}[z, w]$ and such that for every $I \in \mathbb{S}$ the restriction $\left.h\right|_{\mathbb{C}_{I}^{2}}: \mathbb{C}_{I}^{2} \rightarrow \Omega \cap \mathbb{C}_{I}^{2}$ is a biholomorphic map; in other words, $\Omega \cap \mathbb{C}_{I}^{2}$ is a Fatou-Bieberbach domain in $\mathbb{C}_{I}^{2}$.

The main result of this section is the following 
Theorem 3 There exists a slice Fatou-Biebaerbach domain in $\mathbb{H}^{2}$ avoiding $\mathcal{T}$.

We provide the construction of a slice Fatou-Bieberbach domain by adapting the standard procedure of 'escaping points' to the quaternionic setting and using shears as automorphisms with volume 1.

To this end we follow the approximation scheme presented in Lemma 2.1 of [2] and stated here as Lemma 5.

Before describing the approximation scheme in Lemma 5, we need to prove two technical lemmas. We use the following notation. Let $\Delta\left(r_{1}, r_{2}\right):=B^{4}\left(0, r_{1}\right) \times B^{4}\left(0, r_{2}\right)$ be a biball, i.e., the product of two balls centered at the origin with radii $r_{1}, r_{2}>0$. We denote by $\Delta=\Delta(1,1)$ the product of two unit balls; furthermore, if $V$ is a subset of $\mathbb{W}^{2}$ and $\delta$ a positive real number, we define $V+\delta \Delta:=\bigcup_{\left(p_{1}, p_{2}\right) \in V} B^{4}\left(p_{1}, \delta\right) \times B^{4}\left(p_{2}, \delta\right)$.

Lemma 3 Let $U, V$ be two biballs, $U \Subset V$. Then there exists a constant c depending only on $U$ and $V$ such that for any shear $\Phi$ it holds that $|D(\Phi-\mathrm{id})|_{\infty, U}<c|\Phi-\mathrm{id}|_{V}$.

Proof Any shear can be written either the form $\Phi(z, w)=(z, w)+f(z-\mu w)(\mu, 1),(\mu \in \mathbb{R}$, $f \in \mathcal{S R}(\mathbb{W}))$ or $\Phi(z, w)=(z, w)+(f(w), 0), f \in \mathcal{S R}(\mathbb{W})$.

Consider $U=\Delta\left(\varrho_{1}, \varrho_{2}\right), V=\Delta\left(r_{1}, r_{2}\right)$, with $\varrho_{j}<r_{j}, j=1,2$, and define $R_{U}=\varrho_{1}+|\mu| \varrho_{2}$, $R_{V}=r_{1}+|\mu| r_{2}$. Since $U \Subset V$, then $R_{U}<R_{V}$. The Jacobian of a shear $\Phi(z, w)=$ $(z, w)+f(z-\mu w)(\mu, 1)$ is

$$
\begin{aligned}
D \Phi(z, w)[h, k] & =\left[\begin{array}{cc}
h+\mu \hat{\partial}_{z} f(z-\mu w)[h] & \mu \hat{\partial}_{w} f(z-\mu w)[k] \\
\hat{\partial}_{z} f(z-\mu w)[h] & k+\hat{\partial}_{w} f(z-\mu w)[k]
\end{array}\right] \\
& =\left[\begin{array}{cc}
h+\mu \hat{\partial}_{z} f(z-\mu w)[h] & -\mu^{2} \hat{\partial}_{z} f(z-\mu w)[k] \\
\hat{\partial}_{z} f(z-\mu w)[h] & k-\mu \hat{\partial}_{z} f(z-\mu w)[k]
\end{array}\right],
\end{aligned}
$$

since for functions of the form $f(z-\mu w)$ with $\mu$ real the chain rule $\hat{\partial}_{w} f(z-\mu w)=$ $-\mu \hat{\partial}_{z} f(z-\mu w$ ) holds (see [8], equation (2.8)). Therefore, we have

$$
\begin{aligned}
|D(\Phi-\mathrm{id})(z, w)|_{\infty}=\max \left\{|\mu|\left|\hat{\partial}_{z} f(z-\mu w)[h]-\mu \hat{\partial}_{z} f(z-\mu w)[k]\right|,\right. \\
\left.\quad\left|\hat{\partial}_{z} f(z-\mu w)[h]-\mu \hat{\partial}_{z} f(z-\mu w)[k]\right|,|h|,|k| \leq 1\right\} \\
\leq(1+|\mu|) \max \left\{\left|\hat{\partial}_{z} f(z-\mu w)[h]-\mu \widehat{\partial}_{z} f(z-\mu w)[k]\right|,|h|,|k| \leq 1\right\} \\
\leq(1+|\mu|) \max \left\{\left|\hat{\partial}_{z} f(z-\mu w)[h]\right|+|\mu|\left|\hat{\partial}_{z} f(z-\mu w)[k]\right|,|h|,|k| \leq 1\right\} \\
\quad \leq(1+|\mu|)^{2} \max \left\{\left|\hat{\partial}_{z} f(z-\mu w)[h]\right|,|h| \leq 1\right\} .
\end{aligned}
$$

Assume that $|\Phi-\mathrm{id}|=\varepsilon$ on $V$. Then $|f(u)| \leq \varepsilon / \sqrt{1+\mu^{2}}=\tilde{\varepsilon}$ for $|u| \leq R_{V}$ and if $(z, w) \in U$ we have $|z-\mu w| \leq R_{U}<R_{V}$. Since $f$ is by definition entire and slice-regular, the coefficients in its power series expansion $f(u)=\sum_{m=0}^{\infty} u^{m} a_{m}$ can be estimated by Cauchy formula, $\left|a_{m}\right| \leq \tilde{\varepsilon} / R_{V}^{m}$. Furthermore, for $(z, w) \in U$, we have

$$
\left|\hat{\partial}_{z}(z-\mu w)^{m}[h]\right| \leq m|h||z-\mu w|^{m-1},
$$

so the following estimate holds 


$$
\begin{aligned}
\left|\hat{\partial}_{z} f(z-\mu w)[h]\right| & \leq \frac{\tilde{\varepsilon}|h|}{R_{V}} \sum_{m=1}^{\infty} m\left|\frac{z-\mu w}{R_{V}}\right|^{m-1} \\
& \leq \frac{\tilde{\varepsilon}|h|}{R_{V}} \sum_{m=1}^{\infty} m\left(\frac{R_{U}}{R_{V}}\right)^{m-1}=\frac{\tilde{\varepsilon}|h| R_{V}}{\left(R_{V}-R_{U}\right)^{2}} .
\end{aligned}
$$

Define $d:=R_{V}\left(R_{V}-R_{U}\right)^{-2}$. The norm $|\cdot|_{\infty}$ of the matrix $D(\Phi(z, w)-(z, w))[h, k]$ for $|h|,|k| \leq 1$ is estimated by

$$
|D(\Phi(z, w)-(z, w))[h, k]|_{\infty} \leq d \tilde{\varepsilon}(1+|\mu|)^{2}=d \varepsilon(1+|\mu|)^{2} / \sqrt{1+\mu^{2}} .
$$

We claim that there exists a constant $c$ depending only on $U$ and $V$ such that $d(1+|\mu|)^{2} / \sqrt{1+\mu^{2}} \leq c$. Indeed, since the function

$$
c(t):=d \frac{(1+t)^{2}}{\sqrt{1+t^{2}}}=\frac{(1+t)^{2}\left(r_{1}+t r_{2}\right)}{\sqrt{1+t^{2}}\left(r_{1}-\varrho_{1}+t\left(r_{2}-\varrho_{2}\right)\right)^{2}}, t \geq 0,
$$

is continuous in $t$, positive and bounded, such a constant exists. Finally, if the shear is of the form $\Phi(z, w)=(z+f(w), w)$, then the constant is $c(\infty)=\frac{r_{2}}{\left(r_{2}-\varrho_{2}\right)^{2}}$.

Let $\left\{U_{n}\right\}_{n \in \mathbb{N}}$ be an exhaustion of $\mathbb{\boxplus}^{2}$ by biballs such that $U_{n} \Subset U_{n+1}$. For each $n$ we define biballs $U_{n}^{\prime \prime \prime} \Subset U_{n}^{\prime \prime} \Subset U_{n}^{\prime}$ by strictly increasing the radii of $U_{n}$, so that we also have $U_{n}^{\prime} \Subset U_{n+1}$, more precisely, we choose a (strictly decreasing) sequence of positive real numbers $\left\{\delta_{n}\right\}_{n \in \mathbb{N}}$ such that $U_{n}^{\prime}:=U_{n}+\delta_{n} \Delta \Subset U_{n}+2 \delta_{n} \Delta \Subset U_{n+1}$. Define $U_{n}^{\prime \prime}:=U_{n}+\left(2 \delta_{n} / 3\right) \Delta$ and $U_{n}^{\prime \prime \prime}:=U_{n}+\left(\delta_{n} / 3\right) \Delta$. Then

$$
U_{n} \Subset U_{n}^{\prime \prime \prime} \Subset U_{n}^{\prime \prime} \Subset U_{n}^{\prime} \Subset U_{n+1}
$$

for every $n \in \mathbb{N}$.

Lemma 4 Let the sequences of sets $\left\{U_{n}\right\}_{n \in \mathbb{N}}$ and $\left\{\delta_{n}\right\}_{n \in \mathbb{N}}$ be as above and let $\left\{\Phi_{n}\right\}_{n \in \mathbb{N}}$ be a sequence of shears. Define $\Psi_{l, m}=\Phi_{l} \circ \ldots \circ \Phi_{m+1}$ for $l>m$ and $\Psi_{m, m}:=\mathrm{id}$. Assume that for every choice of $l>m \geq n$ the following inclusions hold:

$$
\Psi_{l, m}\left(U_{n}\right) \Subset U_{n}^{\prime \prime \prime}, \Psi_{l, m}\left(U_{n}^{\prime \prime \prime}\right) \Subset U_{n}^{\prime \prime}, \Psi_{l, m}\left(U_{n}^{\prime \prime}\right) \Subset U_{n}^{\prime}, \Psi_{l, m}\left(U_{n}^{\prime}\right) \Subset U_{n+1} .
$$

Let a sequence $\left\{\varepsilon_{n}\right\}_{n \in \mathbb{N}}$ of positive real numbers be given such that $\left|\Phi_{n}-\mathrm{id}\right|_{U_{n-1}^{\prime}}<\varepsilon_{n-1}$ for every $n \in \mathbb{N}$. Fix $k>1$ and let $l>m \geq n \geq k$. Then

$$
\left|\Psi_{l, m}-\mathrm{id}\right|_{U_{k}^{\prime \prime}}<\sum_{j=m+1}^{l} \varepsilon_{j-1}
$$

and

$$
\left|D\left(\Psi_{l, m}-\mathrm{id}\right)\right|_{\infty, \Psi_{m, n}\left(U_{k}^{\prime \prime \prime}\right)}<\prod_{j=m+1}^{l}\left(1+c_{j-1} \varepsilon_{j-1}\right)-1
$$

where $c_{j}$ are given by Lemma 3 with respect to sets $U=U_{j}^{\prime \prime}$ and $V=U_{j}^{\prime}$. 
Remark 4 By definition $\Psi_{l, m} \circ \Psi_{m, n}=\Psi_{l, n}$ and $\Psi_{m+1, m}=\Phi_{m+1}$.

Proof For any choice of integers $l>m \geq n \geq k$, the triangle inequality gives

$$
\begin{aligned}
\left|\Psi_{l, m}-\mathrm{id}\right|_{U_{k}^{\prime \prime}} & =\left|\Psi_{l, m}-\Psi_{l-1, m}+\Psi_{l-1, m}-\mathrm{id}\right|_{U_{k}^{\prime \prime}} \\
& \leq\left|\Phi_{l} \circ \Psi_{l-1, m}-\Psi_{l-1, m}\right|_{U_{k}^{\prime \prime}}+\left|\Psi_{l-1, m}-\mathrm{id}\right|_{U_{k}^{\prime \prime}}
\end{aligned}
$$

Because $l>k$, we have the inclusion $U_{k}^{\prime} \subset U_{l-1}^{\prime}$ and using (4) we estimate

$$
\left|\Phi_{l} \circ \Psi_{l-1, m}-\Psi_{l-1, m}\right|_{U_{k}^{\prime \prime}}=\left|\Phi_{l}-\mathrm{id}\right|_{\Psi_{l-1, m}\left(U_{k}^{\prime \prime}\right)} \leq\left|\Phi_{l}-\mathrm{id}\right|_{U_{k}^{\prime}} \leq\left|\Phi_{l}-\mathrm{id}\right|_{U_{l-1}^{\prime}}
$$

and then by induction

$$
\left|\Psi_{l, m}-\mathrm{id}\right|_{U_{k}^{\prime \prime}} \leq \sum_{j=m+1}^{l}\left|\Psi_{j, m}-\Psi_{j-1, m}\right|_{U_{k}^{\prime \prime}} \leq \sum_{j=m+1}^{l}\left|\Phi_{j}-\mathrm{id}\right|_{U_{j-1}^{\prime}}<\sum_{j=m+1}^{l} \varepsilon_{j-1} .
$$

For the second estimate, first observe that $\left|\Phi_{j}-\mathrm{id}\right|_{U_{j-1}^{\prime}}<\varepsilon_{j-1}$ implies $\left|D\left(\Phi_{j}-\mathrm{id}\right)\right|_{\infty, U_{j-1}^{\prime \prime}}$ $<c_{j-1} \varepsilon_{j-1}$ by Lemma 3. Fix $m \geq n \geq k$ and proceed by induction on $l$. For $l=m+1$ we have $\Psi_{m+1, m}=\Phi_{m+1}$, so there is nothing to prove for the base case of induction. Assume that the claim holds true for $l-1 \geq m+1$. By the chain rule for mappings in $\mathcal{H}^{2}[z, w]$ we have

$$
D\left(\Phi_{l} \circ \Psi_{l-1, m}\right)(z, w)[h, k]=D\left(\Phi_{l}\right)\left(\Psi_{l-1, m}(z, w)\right) \diamond D \Psi_{l-1, m}(z, w)[h, k] .
$$

Since the operations $D$ and $\diamond$ are additive and the matrix norm $|\cdot|_{\infty}$ is sub-multiplicative with respect to $\diamond$ (see Sect. 1.1), we can write

$$
\begin{aligned}
D\left(\Psi_{l, m}-\mathrm{id}\right)(z, w)[h, k]= & D\left(\Phi_{l} \circ \Psi_{l-1, m}-\Psi_{l-1, m}\right)(z, w)[h, k] \\
& +D\left(\Psi_{l-1, m}-\mathrm{id}\right)(z, w)[h, k] \\
= & D\left(\Phi_{l}-\mathrm{id}\right)\left(\Psi_{l-1, m}(z, w)\right) \diamond D \Psi_{l-1, m}(z, w)[h, k]+ \\
& +D\left(\Psi_{l-1, m}-\mathrm{id}\right)(z, w)[h, k]
\end{aligned}
$$

and, by induction hypothesis, we have

$$
\begin{aligned}
\left|D\left(\Psi_{l, m}-\mathrm{id}\right)\right|_{\infty, \Psi_{m, n}\left(U_{k}^{\prime \prime \prime}\right)=} & \left|D\left(\Phi_{l}-\mathrm{id}\right)\right|_{\infty, \Psi_{l-1, m}\left(\Psi_{m, n}\left(U_{k}^{\prime \prime \prime}\right)\right)}\left|D \Psi_{l-1, m}\right|_{\infty, \Psi_{m, n}\left(U_{k}^{\prime \prime \prime}\right)} \\
& +\left|D\left(\Psi_{l-1, m}-\mathrm{id}\right)\right|_{\infty, \Psi_{m, n}\left(U_{k}^{\prime \prime \prime}\right)} \\
\leq & \left|D\left(\Phi_{l}-\mathrm{id}\right)\right|_{\infty, \Psi_{l-1, n}\left(U_{k}^{\prime \prime \prime}\right)}\left|D \Psi_{l-1, m}\right|_{\infty, \Psi_{m, n}\left(U_{k}^{\prime \prime \prime}\right)} \\
& +\left|D\left(\Psi_{l-1, m}-\mathrm{id}\right)\right|_{\infty, \Psi_{m, n}\left(U_{k}^{\prime \prime \prime}\right)} \\
< & c_{l-1} \varepsilon_{l-1} \prod_{j=m+1}^{l-1}\left(1+c_{j-1} \varepsilon_{j-1}\right)+\prod_{j=m+1}^{l-1}\left(1+c_{j-1} \varepsilon_{j-1}\right)-1 \\
= & \prod_{j=m+1}^{l}\left(1+c_{j-1} \varepsilon_{j-1}\right)-1,
\end{aligned}
$$

since the inclusions $\Psi_{l-1, n}\left(U_{k}^{\prime \prime \prime}\right) \Subset U_{k}^{\prime \prime} \Subset U_{l-1}^{\prime \prime}$ and the definition of constants $c_{j}$ imply $\left|D\left(\Phi_{l}-\mathrm{id}\right)\right|_{\infty, \Psi_{l-1, n}\left(U_{k}^{\prime \prime \prime}\right)} \leq\left|D\left(\Phi_{l}-\mathrm{id}\right)\right|_{\infty, U_{l-1}^{\prime \prime}}<c_{l-1} \varepsilon_{l-1}$. Furthermore, by induction hypothesis, $\left|D \Psi_{l-1, m}\right|_{\infty, \Psi_{m, n}\left(U_{k}^{\prime \prime \prime}\right)} \leq 1+\left|D \Psi_{l-1, m}-\mathrm{id}\right|_{\infty, \Psi_{m, n}\left(U_{k}^{\prime \prime \prime}\right)}<\prod_{j=m+1}^{l-1}\left(1+c_{j-1} \varepsilon_{j-1}\right)$. 
Lemma 5 Let the sequences $\left\{U_{n}\right\}_{n \in \mathbb{N}},\left\{\delta_{n}\right\}_{n \in \mathbb{N}}$ and $\left\{c_{n}\right\}_{n \in \mathbb{N}}$ be as in Lemma 4 . Let $\left\{\Phi_{n}\right\}_{n \in \mathbb{N}}$ be a sequence of shears and let $\left\{\varepsilon_{n}\right\}_{n \in \mathbb{N}},\left\{\varepsilon_{n}^{\prime}\right\}_{n \in \mathbb{N}}, \varepsilon_{n}, \varepsilon_{n}^{\prime} \in(0,1)$ be strictly decreasing sequences converging to 0 such that $\varepsilon_{n}<\min \left\{\delta_{n}, \log \left(1+\varepsilon_{n}^{\prime}\right)\right\}$ for any $n \in \mathbb{N}$ and such that for any $n \in \mathbb{N}$

(i) $\left|\Phi_{n+1}-\mathrm{id}\right|_{U^{\prime}}<2^{-n} \min \left\{1, c_{n}^{-1}\right\} \varepsilon_{n} / 6$,

(ii) if $\Phi \in \mathcal{C}^{1}\left(U_{n}^{\prime \prime \prime \prime}\right)$ satisfies $|\Phi-\mathrm{id}|_{U_{n}^{\prime \prime \prime}}<\varepsilon_{n}^{\prime}$ and $|D(\Phi-\mathrm{id})|_{\infty, U_{n}^{\prime \prime \prime}}<\varepsilon_{n}^{\prime}$, then $\Phi$ is one to one in $U_{n}$.

For each $n \in \mathbb{N}$ define $\Psi_{n}:=\Phi_{n} \circ \Phi_{n-1} \circ \ldots \circ \Phi_{1}$. Let $\Omega_{n}:=\Psi_{n}^{-1}\left(U_{n}\right)$. Then $\Omega_{n} \Subset \Omega_{n+1}$ and the sequence $\left\{\Psi_{n}\right\}_{n \in \mathbb{N}}$ converges, uniformly on compacta of the open set $\Omega:=\cup_{n=1}^{\infty} \Omega_{n}$, to a map $\Psi \in \mathcal{C}^{1}(\Omega)$ which maps $\Omega$ bijectively onto $\mathbb{H}^{2}$.

Before the proof of the Lemma, we make a couple of remarks.

Remark 5 Observe that for any $U_{n} \Subset U_{n}^{\prime \prime \prime}$ one can always choose a positive $\varepsilon_{n}^{\prime}$ such that if $\Phi \in \mathcal{C}^{1}\left(U_{n}^{\prime \prime \prime}\right)$ approximates the identity in the sense of condition (ii), then it is one to one in $U_{n}$.

Remark 6 The condition $(i)$ in Lemma 5 plays a similar role as condition (i) in Lemma 2.1 of [2]. It not only implies that for $m \geq n$ we have $\left|\Phi_{m+1}-\mathrm{id}\right|_{U_{n}^{\prime}} \leq\left(2^{-m} \varepsilon_{m} / 6\right)$ and hence $\Phi_{m+1}\left(U_{n}^{\prime}\right) \subset\left(2^{-m} \varepsilon_{m} / 6\right) \Delta \Subset U_{n+1} \subset U_{m+1}^{\prime}$, but also the validity of inclusions (4).

Indeed, since $\left|\Phi_{m+2}-\mathrm{id}\right|_{U_{m+1}^{\prime}}<2^{-(m+1)} \varepsilon_{m+1} / 6$, we can estimate

$$
\begin{aligned}
\left|\Psi_{m+2, m}-\mathrm{id}\right|_{U_{n}^{\prime}} & <\left|\Psi_{m+2, m}-\Psi_{m+1, m}+\Phi_{m+1}-\mathrm{id}\right|_{U_{n}^{\prime}} \\
& <\left|\Phi_{m+2}-\mathrm{id}\right|_{\Phi_{m+1}\left(U_{n}^{\prime}\right)}+\left|\Phi_{m+1}-\mathrm{id}\right|_{U_{n}^{\prime}} \\
& <\left|\Phi_{m+2}-\mathrm{id}\right|_{U_{m+1}^{\prime}}+\left|\Phi_{m+1}-\mathrm{id}\right|_{U_{m}^{\prime}} \\
& <2^{-(m+1)} \varepsilon_{m+1} / 6+2^{-m} \varepsilon_{m} / 6<\varepsilon_{m} / 3 \leq \varepsilon_{n} / 3<\delta_{n} / 3,
\end{aligned}
$$

so $\Psi_{m+2, m}\left(U_{n}^{\prime}\right) \subset\left(\delta_{n} / 3\right) \Delta \Subset U_{n+1}$. Hence, inductively, for $l>m \geq n$ the estimate

$$
\left|\Psi_{l, m}-\mathrm{id}\right|_{U_{n}^{\prime}}<\left(\sum_{j=m+1}^{\infty} 2^{-(j-1)} \varepsilon_{j-1} / 6\right)<\varepsilon_{m} / 3<\delta_{n} / 3
$$

holds, which together with the definition of the sequence $\left\{\delta_{n}\right\}_{n \in \mathbb{N}}$ implies the validity of inclusions (4) for $l>m \geq n$.

Remark 7 Let us comment the changes in the formulation of Lemma 5 compared to Lemma 2.1 in [2]. In the complex setting the Cauchy formula for holomorphic functions guarantees that the sup-norm estimates on $U_{n}^{\prime}$ imply $C^{1}$-estimates on $U_{n}^{\prime \prime} \Subset U_{n}^{\prime}$, which is not the case for an arbitrary automorphism $\Psi \in \mathcal{H}^{2}[z, w]$, since those are just real analytic. Such estimates, however, hold for mappings $\Psi-\mathrm{id}$, where $\Psi$ is a shear, as presented in Lemma 3.

For similar reasons we have to add $C^{1}$-estimates to point ( $\left.i i\right)$ of the statement of Lemma 5. The new statement is then a classical result in real analysis: if we write $h=\left(h_{0}, h_{1}, h_{2}, h_{3}\right)$ and $z=\left(z_{0}, z_{1}, z_{2}, z_{3}\right)$ (with respect to the orthonormal basis $(1, i, j, k)$ of $\llbracket$ ) then, since the operator $\widehat{\partial}_{z}$ is additive and real linear in $h$, 


$$
\widehat{\partial}_{z} f(z, w)[h]=\sum_{l=0}^{3} h_{l} \frac{\partial}{\partial z_{l}} f(z, w),
$$

and so the condition $|D(\Phi(z, w)-(z, w))[h, k]|<\varepsilon$ for $|h|,|k| \leq 1$ implies that $\Phi$ approximates the identity in $C^{1}$-sense as a real smooth mapping.

Remark 8 The map $\Psi^{-1}$ is defined in $\mathbb{T}^{2}$ but we cannot argue that it is analytic, because in general it is not a slice-regular map in two variables and after composing slice-regular functions one cannot apply Cauchy estimates. So Theorem 3 is not a straightforward application of Lemma 5.

Proof of Lemma 5 With all the above lemmas the proof of Lemma 5 is now similar to the analogous one in [2].

To prove uniform estimates for the mappings and their derivatives on $\Omega$, choose a compact set $K \subset \Omega$ and $\varepsilon>0$. We are looking for $m_{0}>0$ so that for $l>m \geq m_{0}$ the estimates

$$
\left|\Psi_{l}-\Psi_{m}\right|_{K}<\varepsilon \text { and }\left|D\left(\Psi_{l}-\Psi_{m}\right)\right|_{\infty, K}<\varepsilon
$$

hold.

Define $\Psi_{l, m}$ as in Lemma 4. Notice that the assumptions of Lemma 4 are fulfilled, because by Remark 6, the inclusions (4) hold for all $l>m \geq n$. In particular, $\Psi_{n+1, n}\left(U_{n}^{\prime}\right) \Subset U_{n+1}$, or, equivalently, $U_{n}^{\prime} \Subset \Phi_{n+1}^{-1}\left(U_{n+1}\right)$.

Define $\Omega_{n}^{\prime}:=\Psi_{n}^{-1}\left(U_{n}^{\prime}\right), \Omega_{n}^{\prime \prime}:=\Psi_{n}^{-1}\left(U_{n}^{\prime \prime}\right)$ and $\Omega_{n}^{\prime \prime \prime}:=\Psi_{n}^{-1}\left(U_{n}^{\prime \prime \prime}\right)$. The first three of the following inclusions

$$
\Omega_{n} \Subset \Omega_{n}^{\prime \prime \prime} \Subset \Omega_{n}^{\prime \prime} \Subset \Omega_{n}^{\prime} \Subset \Omega_{n+1}
$$

are obvious. The last inclusion $\Omega_{n}^{\prime} \Subset \Omega_{n+1}$ is equivalent to

$$
\Psi_{n+1}\left(\Omega_{n}^{\prime}\right) \Subset \Psi_{n+1}\left(\Omega_{n+1}\right)=U_{n+1} .
$$

Because $\Psi_{n+1}\left(\Omega_{n}^{\prime}\right)=\Phi_{n+1}\left(\Psi_{n}\left(\Omega_{n}^{\prime}\right)\right)=\Phi_{n+1}\left(U_{n}^{\prime}\right)$ the inclusion (8) is equivalent to $U_{n}^{\prime} \Subset \Phi_{n+1}^{-1}\left(U_{n+1}\right)$ which holds true as mentioned. As a consequence we have that $\left\{\Omega_{n}\right\}_{n \in \mathbb{N}}$ is an exhaustion of $\Omega=\cup_{n} \Omega_{n}$.

Notice that also

$$
\Psi_{m}\left(\Omega_{n}^{\prime \prime \prime}\right)=\Psi_{m, n}\left(U_{n}^{\prime \prime \prime}\right) \Subset U_{n}^{\prime \prime}
$$

holds for every $m \geq n$.

Fix $n_{0} \in \mathbb{N}$ such that $K \subset \Omega_{n_{0}}$. Hence it suffices to show that the sequence $\left\{\Psi_{m}\right\}_{m \in \mathbb{N}}$ converges uniformly in $\mathcal{C}^{1}$ sense on $\Omega_{n}$ for some $n \geq n_{0}$. Let $l>m \geq n \geq n_{0}$. Because of the inclusions in (9) we have

$$
\left|\Psi_{l}-\Psi_{m}\right|_{\Omega_{n}^{\prime \prime \prime}}=\left|\Psi_{l, m}-\mathrm{id}\right|_{\Psi_{m}\left(\Omega_{n}^{\prime \prime \prime}\right)} \leq\left|\Psi_{l, m}-\mathrm{id}\right|_{U_{n}^{\prime \prime}}
$$

and by Lemma 4 we estimate

$$
\left|\Psi_{l}-\Psi_{m}\right|_{\Omega_{n}^{\prime \prime \prime}} \leq \sum_{j=m}^{l-1}\left|\Phi_{j+1}-\mathrm{id}\right|_{U_{n}^{\prime \prime}} \leq \sum_{j=n}^{\infty} 2^{-j} \varepsilon_{j} / 6<2^{-n} \varepsilon_{n} / 3<\varepsilon
$$

for large enough $n$. 
The estimate for the derivative is a bit more delicate.

Since the norm $|\cdot|_{\infty}$ is sub-multiplicative and the derivative is additive, we have the following estimate

$$
\begin{aligned}
& \left|D\left(\Psi_{l}-\Psi_{m}\right)\right|_{\infty, \Omega_{n}^{\prime \prime \prime}} \leq\left|D\left(\Psi_{l, m}-\mathrm{id}\right)\right|_{\infty, \Psi_{m}\left(\Omega_{n}^{\prime \prime \prime}\right)} \cdot\left|D \Psi_{m}\right|_{\infty, \Omega_{n}^{\prime \prime \prime}} \\
& =\left|D\left(\Psi_{l, m}-\mathrm{id}\right)\right|_{\infty, \Psi_{m, n}\left(U_{n}^{\prime \prime \prime}\right)} \cdot\left|D \Psi_{m}\right|_{\infty, \Omega_{n}^{\prime \prime \prime}}
\end{aligned}
$$

The assumptions of Lemma 4 are now fulfilled and so we can estimate the term $\left|D\left(\Phi_{l, m}-\mathrm{id}\right)\right|_{\infty, \Psi_{m, n}\left(U_{n}^{\prime \prime \prime}\right)}$ in (12) by inserting $2^{-j+1} \min \left\{1, c_{j-1}^{-1}\right\} \varepsilon_{j-1} / 6$ instead of $\varepsilon_{j-1}$ in (6) and using $c_{j-1} \min \left\{1, c_{j-1}^{-1}\right\} \leq 1$ to obtain

$$
\begin{aligned}
\left|D\left(\Psi_{l, m}-\mathrm{id}\right)\right|_{\infty, \Psi_{m, n}\left(U_{n}^{\prime \prime \prime}\right)} & \leq \prod_{j=m+1}^{\infty}\left(1+2^{-j+1} \varepsilon_{j-1} / 6\right)-1 \\
& \leq e^{\sum_{j=m+1}^{\infty} 2^{-j+1} \varepsilon_{j-1} / 6}-1 \leq e^{2^{-m} \varepsilon_{m} / 3}-1 \\
& <\varepsilon_{m}^{\prime} \leq \varepsilon_{n}^{\prime}<\varepsilon
\end{aligned}
$$

for large enough $n \geq n_{0}$. We also observe that

$$
0<e^{2^{-n} \varepsilon_{n} / 3}-1<2 \text { for } n \geq 1
$$

since $\varepsilon_{n}<1$.

In order to estimate $\left|D \Psi_{m}\right|_{\infty, \Omega^{\prime \prime \prime}}$ we first fix $n$ so that the estimates (11) and (13) hold. The mapping $\Psi_{n}$ is entire, the set $\Omega_{n}^{\prime \prime}$ relatively compact, so $\left|D \Psi_{n}\right|_{\infty, \Omega_{n}^{\prime \prime \prime}}<M$ for some positive constant $M$.

Taking into account that $\Psi_{n}\left(\Omega_{n}^{\prime \prime \prime}\right)=U_{n}^{\prime \prime \prime}=\Psi_{n, n}\left(U_{n}^{\prime \prime \prime}\right)$ we estimate

$$
\begin{aligned}
& \left|D \Psi_{m}\right|_{\infty, \Omega_{n}^{\prime \prime \prime}}=\left|D\left(\Psi_{m}-\Psi_{n}\right)\right|_{\infty, \Omega_{n}^{\prime \prime \prime}}+\left|D \Psi_{n}\right|_{\infty, \Omega_{n}^{\prime \prime \prime}} \\
& \leq\left|D\left(\Psi_{m, n}-\mathrm{id}\right)\right|_{\infty, \Psi_{n}\left(\Omega_{n}^{\prime \prime \prime}\right)}\left|D \Psi_{n}\right|_{\infty, \Omega_{n}^{\prime \prime \prime}}+\left|D \Psi_{n}\right|_{\infty, \Omega_{n}^{\prime \prime \prime}} \\
& \leq\left|D\left(\Psi_{m, n}-\mathrm{id}\right)\right|_{\infty, \Psi_{n, n}\left(U_{n}^{\prime \prime \prime}\right)}\left|D \Psi_{n}\right|_{\infty, \Omega_{n}^{\prime \prime \prime}+\left|D \Psi_{n}\right|_{\infty, \Omega_{n}^{\prime \prime \prime}}} \\
& \leq 2 M+M=3 M \text {, }
\end{aligned}
$$

since by (13) and (14) we have $\left|D\left(\Psi_{m, n}-\mathrm{id}\right)\right|_{\infty, \Psi_{n, n}\left(U_{n}^{\prime \prime \prime}\right)}<2$ for all $m \geq n$. Hence the derivative $D\left(\Psi_{l}-\Psi_{m}\right)$ is by (12) bounded by

$$
\begin{aligned}
\left|D\left(\Psi_{l}-\Psi_{m}\right)\right|_{\infty, \Omega_{n}^{\prime \prime \prime}} & \leq\left|D\left(\Psi_{l, m}-\mathrm{id}\right)\right|_{\infty, \Psi_{m, n}\left(U_{n}^{\prime \prime \prime}\right)} \cdot\left|D \Psi_{m}\right|_{\infty, \Omega_{n}^{\prime \prime \prime}} \\
& \leq \varepsilon_{m}^{\prime} 3 M .
\end{aligned}
$$

Choose $m_{0}$ large enough so that $\varepsilon_{m_{0}}^{\prime}<\varepsilon /(3 M)$. Then we have

$$
\left|D\left(\Psi_{l}-\Psi_{m}\right)\right|_{\infty, \Omega_{n}} \leq\left|D\left(\Psi_{l}-\Psi_{m}\right)\right|_{\infty, \Omega_{n}^{\prime \prime \prime}}<\varepsilon
$$

as desired.

The sequence $\left\{\Psi_{m}\right\}_{m \in \mathbb{N}}$ thus converges to a map $\Psi$ defined in the open set $\Omega$ uniformly in $\mathcal{C}^{1}$ sense - on compact sets. The limit map $\Psi: \Omega \rightarrow \Psi(\Omega)$ is at least $\mathcal{C}^{1}$ in $\Omega$.

On each $U_{n}^{\prime \prime \prime}$, the map $\Psi \circ \Psi_{n}^{-1}=\lim _{m \rightarrow \infty} \Psi_{m, n}$ satisfies

$$
\left|\Psi \circ \Psi_{n}^{-1}-\mathrm{id}\right|_{U_{n}^{\prime \prime \prime}} \leq \lim _{m \rightarrow \infty}\left|\Psi_{m, n}-\mathrm{id}\right|_{U_{n}^{\prime \prime}} \leq 2^{-n+1} \varepsilon_{n} / 3<\varepsilon_{n}^{\prime}
$$


and

$$
\left|D\left(\Psi \circ \Psi_{n}^{-1}-\mathrm{id}\right)\right|_{\infty, U_{n}^{\prime \prime \prime}} \leq \lim _{m \rightarrow \infty}\left|D\left(\Psi_{m, n}-\mathrm{id}\right)\right|_{\infty, U_{n}^{\prime \prime \prime}} \leq e^{2^{-n} \varepsilon_{n} / 3}-1<\varepsilon_{n}^{\prime}
$$

therefore by (ii) $\Psi \circ \Psi_{n}^{-1}$ is injective in each $U_{n}$, so the map $\Psi$ is injective in $\Omega_{n}$. Therefore, it is injective in $\Omega$; furthermore, by Brouwer's theorem on invariance of domains, the map $\Psi$ is also an open map.

To prove that $\Psi$ is surjective, we will actually show - as in [2] - that $\Psi(\Omega)$ contains all the sets $U_{n}^{\prime \prime}$.

Fix $n$ such that $r_{n-1}:=d\left(\partial U_{n-1}, 0\right)>1$. We claim that $U_{n-1}^{\prime \prime} \Subset \Psi\left(\bar{\Omega}_{n}\right)$. Define $G_{n}:=U_{n-1}^{\prime \prime} \cap \Psi\left(\bar{\Omega}_{n}\right)$ which is a closed set in $U_{n-1}^{\prime \prime}$ because $\Psi\left(\bar{\Omega}_{n}\right)$ is compact (since $\bar{\Omega}_{n}$ is) and therefore closed. By construction $\Psi\left(\bar{\Omega}_{n}\right)=\stackrel{n-1}{\Psi} \Psi_{n}^{-1}\left(\bar{U}_{n}\right)$ and by (10)

$$
\left|\Psi \circ \Psi_{n}^{-1}-\mathrm{id}\right|_{U_{n-1}^{\prime \prime}} \leq 2^{-n+1} \varepsilon_{n} / 3
$$

so $\Psi \circ \Psi_{n}^{-1}(0) \in G_{n}$ because $\left|\Psi \circ \Psi_{n}^{-1}(0)\right|<\varepsilon_{n}<1<r_{n-1}$. For $w \in G_{n}$ we have that $w=\Psi \circ \Psi_{n}^{-1}(z)$ for some $z \in \bar{U}_{n}$ and hence $|w-z|<2^{-n+1} \varepsilon_{n} / 3<\delta_{n} / 3<\delta_{n-1} / 3$, so $z \in U_{n-1}^{\prime \prime}+\Delta \delta_{n-1} / 3 \Subset U_{n-1}^{\prime}$ and there is an open neighborhood $U \subset U_{n-1}^{\prime} \subset U_{n}$ of $z$. Then ${ }^{n-1} \circ \Psi_{n}^{-1}(U) \subset \Psi \circ \Psi_{n}^{-1}\left(U_{n}\right)=\Psi\left(\Omega_{n}\right)$ is an open set containing $w$, ${ }^{n-1}$ since $\Psi \circ \Psi_{n}^{-1}$ is an open mapping. The set $\Psi \circ \Psi_{n}^{-1}(U) \cap U_{n-1}^{\prime \prime}$ is then an open neighborhood of $w$ in $\Psi\left(\Omega_{n}\right) \cap U_{n-1}^{\prime \prime} \subset G_{n}$; in other words, the set $G_{n}$ is a nonempty open and closed subset of $U_{n-1}^{\prime \prime}$ and since the latter is connected, we conclude that $G_{n}=U_{n-1}^{\prime \prime}$.

Remark 9 By the estimate (15), the sequence $\left\{\Psi \circ \Psi_{m}^{-1}\right\}_{m \in \mathbb{N}}$ converges uniformly on compact subsets of $\mathbb{H}^{2}$ to the identity mapping and hence also the sequence $\left\{\Psi_{m}^{-1}=\Phi_{1}^{-1} \circ \ldots \circ \Phi_{m}^{-1}\right\}_{m \in \mathbb{N}}$ converges to $\Psi^{-1}$ uniformly on compact subsets of $\mathbb{T}^{2}$. Consider $\Phi_{n}(z, w)=(z, w)+f_{n}\left(z-\mu_{n} w\right)\left(\mu_{n}, 1\right)$; we assume that each (slice-regular) function $f_{n}$ has a zero of multiplicity $M_{n}$ at the origin, with $M_{n}$ strictly increasing with $n$, and the power series representative (there are infinitely many of them, see [7]) for $f_{n}\left(z-\mu_{n} w\right)$ is the one obtained by formally plugging the term $z-\mu_{n} w$ into power series for $f_{n}$, hence the series expansion for $f_{n}\left(z-\mu_{n} w\right)$ begins with the homogeneous polynomials of degree $M_{n}$ in $(z, w)$. Then the power series expansions of $\left\{\Phi_{1}^{-1} \circ \ldots \circ \Phi_{n}^{-1}\right\}_{n \in \mathbb{N}}$, which are obtained inductively by formally plugging the power series for $\Phi_{n}^{-1}$ into the power series $\Phi_{1}^{-1} \circ \ldots \circ \Phi_{n-1}^{-1}$, converge to a formal power series expansion, since any fixed homogenous polynomial in the power series expansion changes only finitely many times, due to strictly increasing orders of vanishing of the functions $f_{n}$ at the origin. Because of the lack of Cauchy estimates we cannot say that the formal power series is convergent with limit $\Psi^{-1}$.

Proof of Theorem 3 As a first step in the proof, we move the set $\mathcal{T}=\{(2 n-1,0), n \in \mathbb{N}\} \cup\{(2 n+\mathbb{S}, 0), n \in \mathbb{N}\}$ to a more convenient position, namely to the set $\mathcal{T}^{\prime}=\left\{\left(-n^{2},-n^{2}\right), n \in \mathbb{N}\right\} \cup\left\{\left(n^{2} I, n^{2} I\right), n \in \mathbb{N}, I \in \mathbb{S}\right\}$ by a composition of a vertical shear and a horizontal shear. The vertical one is defined as $\Phi_{v}(z, w)=(z, w+f(z))$ with $f$ satisfying the interpolation conditions $f(2 n-1)=-n^{2}, f(2 n+I)=n^{2} I$, and the horizontal one is defined as $\Phi_{h}(z, w)=(z+g(w), w)$ with $g$ satisfying the interpolating conditions $g\left(-n^{2}\right)=-n^{2}-2 n+1, g\left(n^{2} I\right)=n^{2} I-2 n-I$. Both $f$ and $g$ can be chosen to have real coefficients and therefore the shears $\Phi_{h}, \Phi_{v}$ both preserve $\mathbb{C}_{I} \times \mathbb{C}_{I}$ for all $I \in \mathbb{S}$.

Then, for any natural number $m$, we define the subset $\mathcal{T}^{\prime}{ }_{m} \subset \mathcal{T}^{\prime}$ to be the set

$$
\mathcal{T}_{m}{ }^{\prime}=\left\{\left(-n^{2},-n^{2}\right), n \geq m\right\} \cup\left\{\left(n^{2} I, n^{2} I\right), n \geq m, I \in \mathbb{S}\right\} .
$$


and construct two shears $\Phi_{v, m}=\mathrm{id}+\left(0,-f_{m}\right)$ and $\Phi_{h, m}=\mathrm{id}+\left(-g_{m}, 0\right)$, where $f_{m}$ and $g_{m}$ are entire slice-regular functions with nonnegative coefficients in their power series expansions and such that for any $m \in \mathbb{N}$ the map $\Phi_{h, m} \circ \Phi_{v, m}$ has the property $\Phi_{h, m} \circ \Phi_{v, m}\left(\mathcal{T}_{m}^{\prime}\right)=\mathcal{T}_{m+1}^{\prime}$ and $\Phi_{h, m}$ approximates the identity map on compact subsets of $m^{2} \Delta$ whereas $\Phi_{v, m}$ approximates the identity map on compact subsets of $(m+1)^{2} \Delta$.

To this end, for $n \geq m$, we require the following interpolation condition for $f_{m}$ and $g_{m}$ :

$$
\begin{gathered}
f_{m}\left(-n^{2}\right)=2 n+1, f_{m}\left(n^{2} I\right)=-(2 n+1) I, I \in \mathbb{S} \\
g_{m}\left(-(n+1)^{2}\right)=2 n+1, g_{m}\left((n+1)^{2} I\right)=-(2 n+1) I, I \in \mathbb{S},
\end{gathered}
$$

together with the property that $f_{m}$ is as small as we wish on compact subsets of $B^{4}\left(0, m^{2}\right) \subset \mathbb{H}$ and $g_{m}$ is as small as we wish on compact subsets of $B^{4}\left(0,(m+1)^{2}\right) \subset \mathbb{H}$, both with the order of vanishing at 0 as large as we wish.

We will actually look for such maps in the form $f_{m}:=f_{m}^{P}+f_{m}^{S}$ and $g_{m}:=g_{m}^{P}+g_{m}^{S}$, where the maps with superscript $P$ interpolate on points and leave the spheres fixed and the maps with superscript $S$ interpolate on spheres and leave points fixed.

For each $n \in \mathbb{N}$, consider

$$
\tilde{\phi}_{n}^{P}(z):=\prod_{k \neq n}\left(1+\frac{z}{k^{2}}\right)^{2} \prod_{k=1}^{\infty}\left(1+\frac{z^{2}}{k^{4}}\right),
$$

and

$$
\phi_{n}^{P}(z):=\frac{\tilde{\phi}_{n}^{P}(z)}{\tilde{\phi}_{n}^{P}\left(-n^{2}\right)} .
$$

We have

$$
\phi_{n}^{P}\left(-n^{2}\right)=1, \phi_{n}^{P}\left(-k^{2}\right)=0 \text { if } k \neq n, \text { and } \phi_{n}^{P}\left(m^{2} I\right)=0, \forall m \in \mathbb{N}, \forall I \in \mathbb{S} .
$$

For a suitable choice of an increasing sequence $\left\{M_{n}\right\}_{n \in \mathbb{N}}$, the function

$$
f_{m}^{P}(z):=\sum_{n=m}^{\infty}\left(\frac{z}{n^{2}}\right)^{2 M_{n}} \phi_{n}^{P}(z)(2 n+1)
$$

is well defined and satisfies the interpolation conditions $f_{m}^{P}\left(-n^{2}\right)=2 n+1$ if $n \geq m$ and $f_{m}^{P}\left(k^{2} I\right)=0 \forall k$. Furthermore the coefficients of the power series expansions of $f_{m}^{P}$ are nonnegative, $f_{m}^{P}$ is as small as we wish on compact subsets of $B^{4}\left(0, m^{2}\right)$ with order of vanishing at 0 at least $2 M_{m}$.

In order to define $f_{m}^{S}$, we first consider

$$
\tilde{\phi}(z):=\prod_{k=1}^{\infty}\left(1+\frac{z}{k^{2}}\right), \tilde{\phi}_{n}(z):=\prod_{k \neq n}\left(1+\frac{z^{2}}{k^{4}}\right)^{2} .
$$

We have

$$
\tilde{\phi}\left(-k^{2}\right)=0 \forall k, \tilde{\phi}_{n}\left(k^{2} I\right)=0, \forall k \neq n, \forall I \in \mathbb{S} .
$$


The value $\tilde{\phi}_{n}\left(n^{2} I\right)$ is positive and does not depend on $I \in \mathbb{S}$. Nonetheless $\tilde{\phi}\left(n^{2} I\right)=a+I b \neq 0$ is not necessarily a real number. We are looking for a function of the form

$$
\tilde{\psi}_{n}(z)=\left(\frac{z}{n^{2}}\right)^{l+1}(\alpha z+\beta), \text { with } \alpha, \beta \geq 0, l \in\{0,1,2,3\},
$$

such that

$$
\tilde{\psi}_{n}\left(n^{2} I\right)(a+I b)=-I .
$$

Choose $l \in\{0,1,2,3\}$ so that for $z_{0}=-I^{l}(a+I b)$ we have $\mathfrak{R} e\left(z_{0}\right) \geq 0$ and $\mathfrak{\Im} m\left(z_{0}\right) \leq 0$. Then $\alpha, \beta \in \mathbb{R}$ determined by the equality

$$
\alpha n^{2} I+\beta=\frac{\bar{z}_{0}}{\left|\bar{z}_{0}\right|^{2}}
$$

are nonnegative. Finally, define

$$
\phi_{n}^{S}(z):=\tilde{\psi}_{n}(z) \tilde{\phi}(z) \tilde{\phi}_{n}(z)\left(\tilde{\phi}_{n}\left(n^{2} I\right)\right)^{-1}
$$

for some (and thus for all) $I \in \mathbb{S}$. The coefficients in the power series expansion of $\phi_{n}^{S}$ are nonnegative as desired and, furthermore,

$$
\phi_{n}^{S}\left(n^{2} I\right)=-I, \quad \phi_{n}^{S}\left(k^{2} I\right)=0, \forall k \neq n, \forall I \in \mathbb{S}
$$

and

$$
\phi_{n}^{S}\left(-k^{2}\right)=0, \forall k \in \mathbb{N} .
$$

For a suitable choice of an increasing sequence $\left\{M_{n}\right\}_{n \in \mathbb{N}}$, the function

$$
f_{m}^{S}(z):=\sum_{n=m}^{\infty}\left(\frac{z}{n^{2}}\right)^{4 M_{n}} \phi_{n}^{S}(z)(2 n+1)
$$

is well defined with nonnegative coefficients in its power series expansion, as small as we wish on compact subsets of $B^{4}\left(0, m^{2}\right)$ with order of vanishing at 0 at least $4 M_{m}$ and satisfies the interpolation conditions

$$
f_{m}^{S}\left(n^{2} I\right)=-(2 n+1) I, \quad \forall n \geq m, f_{m}^{S}\left(-k^{2}\right)=0, \forall k .
$$

Then the function $f_{m}:=f_{m}^{P}+f_{m}^{S}$ satisfies all the conditions required, namely $f_{m}$ is as small as we wish on compact subsets of $B^{4}\left(0, \mathrm{~m}^{2}\right)$ with order of vanishing at 0 as large as we wish, has nonnegative coefficients in its power series expansion and fulfills the interpolation conditions (16).

The shear $\Phi_{v, m}(z, w)=\left(z, w-f_{m}(z)\right)$ maps $\left(-n^{2},-n^{2}\right)$ to $\left(-n^{2},-(n+1)^{2}\right)$ and $\left(n^{2} I, n^{2} I\right)$ to $\left(n^{2} I,(n+1)^{2} I\right)$ for $n \geq m, I \in \mathbb{S}$ and approximates the identity on compact subsets of $m^{2} \Delta$ as well as we wish. Also $\Phi_{v, m}^{-1}(z, w)=\left(z, w+f_{m}(z)\right)$ approximates the identity on compact subsets of $m^{2} \Delta$ as well as $\Phi_{v, m}$ does and in addition it has nonnegative coefficients in its power series expansion.

In a similar manner we obtain the shear $\Phi_{h, m}(z, w)=\left(z-g_{m}(w), w\right)$ so that it maps $\left(-n^{2},-(n+1)^{2}\right)$ to $\left(-(n+1)^{2},-(n+1)^{2}\right),\left(n^{2} I,(n+1)^{2} I\right)$ to $\left((n+1)^{2} I,(n+1)^{2} I\right)$ for 
$n \geq m, I \in \mathbb{S}$ and approximates the identity on compact subsets of $(m+1)^{2} \Delta$ as well as we wish.

To define the function $g_{m}^{P}$, for each $n \in \mathbb{N}$, consider a suitable choice of an increasing sequence $\left\{M_{n}\right\}_{n \in \mathbb{N}}$, and put

$$
g_{m}^{P}(z):=\sum_{n=m}^{\infty}\left(\frac{z}{(n+1)^{2}}\right)^{2 M_{n}} \phi_{n+1}^{P}(z)(2 n+1) .
$$

The function $g_{m}^{P}$ is well defined, satisfies the interpolation conditions $g_{m}^{P}\left(-(n+1)^{2}\right)=2 n+1$, for $n \geq m$ and $g_{m}^{P}\left(k^{2} I\right)=0 \forall k$; it has nonnegative coefficients in its power series expansion, is as small as we wish on compact subsets of $B\left(0,(m+1)^{2}\right)$ and with order of vanishing at 0 at least $2 M_{m}$.

To define $g_{m}^{S}$, we recall that

$$
\tilde{\phi}\left(-k^{2}\right)=0 \forall k, \tilde{\phi}_{n+1}\left(k^{2} I\right)=0, \forall k \neq n+1, \forall I \in \mathbb{S} .
$$

Again, the value $\tilde{\phi}_{n+1}\left((n+1)^{2} I\right)$ is positive and does not depend on $I \in \mathbb{S}$ but $\tilde{\phi}\left((n+1)^{2} I\right)=a+I b \neq 0$ is not necessarily a real number.

As in the previous case, we construct a function of the form

$$
\tilde{\psi}_{n+1}(z)=\left(\frac{z}{(n+1)^{2}}\right)^{l+1}(\alpha z+\beta), \quad \text { with } \alpha, \beta \geq 0, l \in\{0,1,2,3\},
$$

such that

$$
\tilde{\psi}_{n+1}\left((n+1)^{2} I\right)(a+I b)=-I .
$$

Let

$$
\phi_{n+1}^{S}(z):=\tilde{\psi}_{n+1}(z) \tilde{\phi}(z) \tilde{\phi}_{n+1}(z)\left(\tilde{\phi}_{n+1}\left((n+1)^{2} I\right)\right)^{-1}
$$

for some (and thus for all) $I \in \mathbb{S}$. The coefficients of the power series expansion of $\phi_{n}^{S}$ are nonnegative; furthermore,

$$
\phi_{n+1}^{S}\left((n+1)^{2} I\right)=-I, \quad \phi_{n+1}^{S}\left(k^{2} I\right)=0, \forall k \neq n+1, \forall I \in \mathbb{S}
$$

and

$$
\phi_{n+1}^{S}\left(-k^{2}\right)=0 \forall k
$$

For a suitable choice of a strictly increasing sequence $\left\{M_{n}\right\}_{n \in \mathbb{N}}$, the function

$$
g_{m}^{S}(z):=\sum_{n=m}^{\infty}\left(\frac{z}{(n+1)^{2}}\right)^{4 M_{n}} \phi_{n+1}^{S}(z)(2 n+1)
$$

is well defined and satisfies the interpolation conditions

$$
g_{m}^{S}\left((n+1)^{2} I\right)=-(2 n+1) I, \quad \forall n \geq m, g_{m}^{S}\left(-k^{2}\right)=0, \forall k ;
$$

it has nonnegative coefficients in its power series expansion, is as small as we wish on compact subsets of $B\left(0,(m+1)^{2}\right)$ and the order of vanishing at 0 is at least $4 M_{m}$. 
Then the function $g_{m}:=g_{m}^{P}+g_{m}^{S}$ satisfies all the conditions required, namely $g_{m}$ is as small as we wish on compact subsets of $B^{4}\left(0,(m+1)^{2}\right)$, has the order of vanishing at 0 as large as we wish and fulfills the interpolation conditions (17).

The shear $\Phi_{h, m}(z, w)=\left(z-g_{m}(w), w\right)$ maps $\left(-n^{2},-(n+1)^{2}\right)$ to $\left(-(n+1)^{2},-(n+1)^{2}\right)$ and $\left(n^{2} I,(n+1)^{2} I\right)$ to $\left((n+1)^{2} I,(n+1)^{2} I\right)$ for $n \geq m, I \in \mathbb{S}$ and approximates the identity on compact subsets of $(m+1)^{2} \Delta$ as well as we wish. Also $\Phi_{h, m}^{-1}(z, w)=\left(z+g_{m}(w), w\right)$ approximates the identity on compact subsets of $(m+1)^{2} \Delta$ as well as $\Phi_{h, m}$ does and in addition it has nonnegative coefficients in its power series expansion.

We now define the sets $U_{m}$ and relabel the shears $\Phi_{v, m}$ and $\Phi_{h, m}$ accordingly. For $m \in \mathbb{N}$ define

$$
\begin{array}{ll}
U_{2 m-1}:=m^{2} \Delta, & \Phi_{2 m-1}:=\Phi_{v, m}, \\
U_{2 m}:=\left((m+1)^{2}-1\right) \Delta, & \Phi_{2 m}:=\Phi_{h, m} .
\end{array}
$$

Choose a strictly decreasing sequence of positive numbers $\left\{\delta_{m}\right\}_{m \in \mathbb{N}}$ such that $U_{m}^{\prime}:=U_{m}+$ $\delta_{m} \Delta \Subset U_{m}+2 \delta_{m} \Delta \Subset U_{m+1}$. Define $U_{m}^{\prime \prime}:=U_{m}+\left(2 \delta_{m} / 3\right) \Delta$ and $U_{m}^{\prime \prime \prime}:=U_{m}+\left(\delta_{m} / 3\right) \Delta$, so

$$
U_{m} \Subset U_{m}^{\prime \prime \prime} \Subset U_{m}^{\prime \prime} \Subset U_{m}^{\prime} \Subset U_{m+1}
$$

for every $m \in \mathbb{N}$.

Let the sequence $\left\{c_{m}\right\}_{m \in \mathbb{N}}$ be given by Lemma 3 with respect to pairs of sets $U=U_{m}^{\prime \prime}$ and $V=U_{m}^{\prime}$.

Choose a strictly decreasing sequence $\left\{\varepsilon_{m}^{\prime}\right\}_{m \in \mathbb{N}}$ of positive numbers converging to 0 so that the condition (ii) of Lemma 5 holds (see also Remark 5).

Then choose a strictly decreasing sequence of positive real numbers $\left\{\varepsilon_{m}\right\}_{m \in \mathbb{N}}$ converging to 0 such that the condition $\varepsilon_{m}<\min \left\{\delta_{m}, \log \left(1+\varepsilon_{m}^{\prime}\right)\right\}$ from Lemma 5 holds for any $m \in \mathbb{N}$.

Choose the sequence of shears $\left\{\Phi_{m}\right\}_{m \in \mathbb{N}}$ which approximate the identity so well, that the assumptions $(i)$ and (ii) of Lemma 5 are fulfilled. Moreover, we require that the orders $M_{m}$ of vanishing at $(0,0)$ of $\Phi_{m}-$ id form a strictly increasing sequence with $M_{m}>m$, so $\Phi_{m}(0,0)=(0,0)$ for all $m \in \mathbb{N}$.

By Lemma 5, the sequence $\left\{\Psi_{m}=\Phi_{m} \circ \ldots \circ \Phi_{1}\right\}_{m \in \mathbb{N}}$ converges on compact subset of $\Omega$ to a diffeomorphism $\Psi: \Omega \rightarrow \mathbb{Z}^{2}$. Since for all $m \in \mathbb{N}$ and $I \in \mathbb{S}$ we have $\left|\Psi_{m}\left(-n^{2},-n^{2}\right)\right| \rightarrow+\infty$ and $\left|\Psi_{m}\left(n^{2} I, n^{2} I\right)\right| \rightarrow+\infty$, as $m \rightarrow+\infty$, the set $\mathcal{T}^{\prime}$ is not contained in $\Omega$.

The limit map $\Psi$ as well as it inverse $\Psi^{-1}$ are limits of mappings, which preserve complex subspaces $\mathbb{C}_{I}^{2}$. Therefore, we have, for any fixed $I \in \mathbb{S}$ and a set $\Omega_{I}:=\Omega \cap \mathbb{C}_{I}^{2} \ni(0,0)$, that

$$
\left.\Psi\right|_{\Omega_{I}}: \Omega_{I} \rightarrow \mathbb{C}_{I}^{2} \text { and }\left.\Psi^{-1}\right|_{\mathbb{C}_{I}^{2}}: \mathbb{C}_{I}^{2} \rightarrow \mathbb{C}_{I}^{2},
$$

so $\left.\Psi\right|_{\Omega_{I}}=\mathbb{C}_{I}^{2}$ and $\Psi_{\Omega_{I}}^{-1}=\left(\left.\Psi\right|_{\mathbb{C}_{I}^{2}}\right)^{-1}$, since $\Psi$ is surjective. As the restrictions of $\left.\Psi_{m}\right|_{\Omega_{I}}$ converge uniformly on compact subsets of $\Omega_{I}$, the limit mappings $\left.\Psi\right|_{\Omega_{I}}$ are holomorphic, and hence $\Omega_{I}$ is a Fatou-Bieberbach domain for any $I \in \mathbb{S}$.

To conclude that $\Omega$ is a slice Fatou-Bieberbach domain, we have to show that $\Psi^{-1}: \mathbb{H}^{2} \rightarrow \Omega$ is real analytic.

By construction, any shear $\Phi_{k}^{-1}$ is a diffeomorphism with nonnegative real coefficients in its power series expansion and the orders of vanishing at $(0,0)$ of $\Phi_{k}^{-1}-$ id form a strictly increasing sequence, so by Remark 9 the formal power series expansion of 
$\Psi^{-1}=\lim _{m \rightarrow+\infty} \Psi_{m}^{-1}$ exists and has nonnegative coefficients. Then, according to [7], for any $m \in \mathbb{N}$ one can write ${ }^{1}$

$$
\Psi_{m}^{-1}(z, w)=\sum_{\alpha \in \Lambda}(z, w)^{\alpha} r_{\alpha, m}, \quad r_{\alpha, m} \in \mathbb{R}, r_{\alpha, m} \geq 0,
$$

which converges absolutely and uniformly on compact subsets of $\mathbb{M}{ }^{2}$. Put $\Lambda^{m}=\cup_{d=0}^{m} \Lambda_{d}$ for $m \in \mathbb{N}$. For any given $\alpha \in \Lambda$, the sequence $r_{\alpha, m}, m \in \mathbb{N}$ is increasing and there exists $m_{0} \in \mathbb{N}$ such that, for $m \geq m_{0}, r_{\alpha, m}=r_{\alpha, m_{0}}=: r_{\alpha}$. Even more, by assumption that the orders of vanishing of $\Psi_{m}^{-1}-\mathrm{id}$ form a strictly increasing sequence, we have $r_{\alpha, m}=r_{\alpha, m_{0}}=: r_{\alpha}$ for every $\alpha \in \Lambda^{m_{0}}$ and $m \geq m_{0}$. In particular, $r_{\alpha, m}=r_{\alpha}$ for any $\alpha \in \Lambda^{m}$.

The formal power series for $\Psi^{-1}$ is

$$
\sum_{\alpha \in \Lambda}(z, w)^{\alpha} r_{\alpha}, \quad r_{\alpha} \in \mathbb{R}, r_{\alpha} \geq 0
$$

which converges absolutely and uniformly on compact subsets of $\mathbb{C}_{I}^{2}$ to $\left.\Psi^{-1}\right|_{\mathbb{C}_{I}^{2}}$ with the series expansion

$$
\left.\Psi^{-1}\right|_{\mathbb{C}_{I}^{2}}=\sum_{p, q=0}^{\infty} z^{p} w^{q} \rho_{p, q} .
$$

In this case, the restriction to $\mathbb{C}_{I}^{2}$ of the formal power series for $\Psi^{-1}$ is convergent and the variables commute, so we have

$$
\rho_{p, q}=\sum_{\alpha \in \Lambda_{p+q},|\alpha|=p} r_{\alpha}
$$

since the coefficients $r_{\alpha}$ are nonnegative, we have $\rho_{p, q} \geq 0$ for all $p, q \in \mathbb{N}_{0}$. For any index subset $\Lambda_{1} \subset \Lambda$ and

$$
S_{\Lambda_{1}}(z, w)=\sum_{\alpha \in \Lambda_{1}}(z, w)^{\alpha} r_{\alpha}
$$

the following estimate holds

$$
\left|S_{\Lambda_{1}}(z, w)\right| \leq \sum_{\alpha \in \Lambda_{1}}\left|(z, w)^{\alpha}\right| r_{\alpha}=S_{\Lambda_{1}}(|z|,|w|) \leq\left.\Psi^{-1}\right|_{\mathbb{C}_{I}^{2}}(|z|,|w|)=\Psi^{-1}(|z|,|w|) .
$$

Fix a compact set $K \subset \mathbb{H}$ and $\varepsilon>0$. We claim that there exists $m_{0} \in \mathbb{N}$ so that if $m \geq m_{0}$ we have $\left|\Psi^{-1}(z, w)-S_{\Lambda^{m}}(z, w)\right|<\varepsilon$ on $K$. By uniform convergence on compacts, there exists $m_{0}$ so that if $m \geq m_{0}$ we have $\left|\Psi^{-1}(z, w)-\Psi_{m}^{-1}(z, w)\right|<\varepsilon / 2$ on $K$. Let $m_{0}$ be so large that $\sum_{p+q \geq m_{0}}^{\infty}|z|^{p}|w|^{q} \rho_{p, q}<\varepsilon / 2$ on $K$. Since for any $m$ we have $r_{\alpha}=r_{\alpha, m}$ if $\alpha \in \Lambda^{m}$, thus for $m \geq m_{0}$

\footnotetext{
${ }^{1}$ Define $\Lambda$ to be a subset of all finite sequences of 0 -s and 1-s, together with the empty sequence, $\Lambda:=\cup_{0}^{\infty} \Lambda_{d}, \quad \Lambda_{d}=\{0,1\}^{d}, \quad d \in \mathbb{N}_{0}$. Given the multiindex $\alpha=\left(\alpha_{1}, \ldots, \alpha_{d}\right) \in\{0,1\}^{d}$, we put $|\alpha|=\alpha_{1}+\ldots+\alpha_{d}$ and

We define $(z, w)^{\emptyset}=1$.

$$
(z, w)^{\alpha}:=\left(z^{\alpha_{1}} w^{1-\alpha_{1}}\right) \cdot \ldots \cdot\left(z^{\alpha_{d}} w^{1-\alpha_{d}}\right) .
$$
}




$$
\begin{aligned}
& \left|\Psi_{m}^{-1}(z, w)-\sum_{\alpha \in \Lambda^{m}}(z, w)^{\alpha} r_{\alpha}\right|=\left|\sum_{\alpha \in \Lambda}(z, w)^{\alpha} r_{\alpha, m}-\sum_{\alpha \in \Lambda^{m}}(z, w)^{\alpha} r_{\alpha}\right| \\
& =\left|\sum_{\alpha \in\left(\Lambda \backslash \Lambda^{m}\right)}(z, w)^{\alpha} r_{\alpha, m}-\sum_{\alpha \in \Lambda^{m}}(z, w)^{\alpha}\left(r_{\alpha}-r_{\alpha, m}\right)\right| \\
& =\left|\sum_{\alpha \in\left(\Lambda \backslash \Lambda^{m}\right)}(z, w)^{\alpha} r_{\alpha, m}\right| \leq \sum_{\alpha \in\left(\Lambda \backslash \Lambda^{m}\right)}(|z|,|w|)^{\alpha} r_{\alpha, m} \\
& \leq \sum_{\alpha \in\left(\Lambda \backslash \Lambda^{m}\right)}(|z|,|w|)^{\alpha} r_{\alpha} \leq \sum_{p+q>m_{0}}^{\infty}|z|^{p}|w|^{q} \rho_{p, q}<\varepsilon / 2,
\end{aligned}
$$

so on $K$ the estimate

$$
\left|\Psi^{-1}(z, w)-S_{\Lambda^{m}}(z, w)\right| \leq\left|\Psi^{-1}(z, w)-\Psi_{m}^{-1}(z, w)\right|+\left|\Psi_{m}^{-1}(z, w)-\sum_{\alpha \in \Lambda^{m}}(z, w)^{\alpha} r_{\alpha}\right|<\varepsilon
$$

holds. The formal series of $\Psi^{-1}$ thus converges absolutely and uniformly on compact subsets of $\mathbb{\boxplus}^{2}$ to $\Psi^{-1}$.

The mapping $F=\Phi_{v}^{-1} \circ \Phi_{h}^{-1} \circ \Psi^{-1} \circ \Phi_{h} \circ \Phi_{v}$ is real analytic and the image $F\left(\mathbb{H}^{2}\right)$ misses the set $\mathcal{T}$. As the shears $\Phi_{h}, \Phi_{v}$ both have real coefficients and thus preserve $\mathbb{C}_{I}^{2}$ for all $I$, the set $F\left(\mathbb{H}^{2}\right)$ is a slice Fatou-Bieberbach domain.

Funding Open access funding provided by Università degli Studi di Trieste within the CRUI-CARE Agreement.

Open Access This article is licensed under a Creative Commons Attribution 4.0 International License, which permits use, sharing, adaptation, distribution and reproduction in any medium or format, as long as you give appropriate credit to the original author(s) and the source, provide a link to the Creative Commons licence, and indicate if changes were made. The images or other third party material in this article are included in the article's Creative Commons licence, unless indicated otherwise in a credit line to the material. If material is not included in the article's Creative Commons licence and your intended use is not permitted by statutory regulation or exceeds the permitted use, you will need to obtain permission directly from the copyright holder. To view a copy of this licence, visit http://creativecommons.org/licenses/by/4.0/.

\section{References}

1. Andrist, R., Ugolini, R.: A new notion of Tameness. J. Math. Anal. Appl. 472(1), 196-215 (2019). https://doi.org/10.1007/978-1-4613-0195-0

2. Globevnik, J.: On Fatou-Bieberbach domains. Math. Z. 229, 91-106 (1998)

3. Gentili, G., Stoppato, C., Struppa, D.: Regular functions of a quaternionic variable. Springer monographs in mathematics. Springer, Heidelberg (2013)

4. Gentili, G., Struppa, D.: A new theory of regular functions of a quaternionic variable. Adv. Math. 216, 279-301 (2007)

5. Gentili, G., Vignozzi, I.: The Weierstrass factorization theorem for slice-regular functions over the quaternions. Ann. Glob. Anal. Geom. 40(4), 435-466 (2011)

6. Ghiloni, R., Perotti, A.: Slice-regular functions of several Clifford variables. In: Proceedings of ICNPAA 2012-Workshop 'Clifford algebras, Clifford analysis and their applications', AIP Conference Processing 1493, 734-738 (2012)

7. Prezelj, J., Vlacci, F.: Divergence zero quaternionic vector fields and Hamming graphs. Ars Math. Contemp. 19(2), 189-208 (2020). https://doi.org/10.26493/1855-3974.2033.974 
8. Prezelj, J., Vlacci, F.: On a class of automorphisms in $\mathbb{H}^{2}$ which resemble the property of preserving volume. Math. Nachr. 294(4), 815-835 (2021). https://doi.org/10.1002/mana.201900259

9. Rosay, J.P., Rudin, W.: Holomorphic Maps from $\mathbb{C}^{n}$ to $\mathbb{C}^{n}$. Trans. AMS 310(1), 47-86 (1988)

Publisher's Note Springer Nature remains neutral with regard to jurisdictional claims in published maps and institutional affiliations. 